\title{
MATHEMATICAL PROGRAMS WITH CARDINALITY CONSTRAINTS: REFORMULATION BY COMPLEMENTARITY-TYPE CONDITIONS AND A REGULARIZATION METHOD*
}

\author{
OLEG P. BURDAKOV ${ }^{\dagger}$, CHRISTIAN KANZOW $^{\ddagger}$, AND ALEXANDRA SCHWARTZ ${ }^{\S}$
}

\begin{abstract}
Optimization problems with cardinality constraints are very difficult mathematical programs which are typically solved by global techniques from discrete optimization. Here we introduce a mixed-integer formulation whose standard relaxation still has the same solutions (in the sense of global minima) as the underlying cardinality-constrained problem; the relation between the local minima is also discussed in detail. Since our reformulation is a minimization problem in continuous variables, it allows us to apply ideas from that field to cardinality-constrained problems. Here, in particular, we therefore also derive suitable stationarity conditions and suggest an appropriate regularization method for the solution of optimization problems with cardinality constraints. This regularization method is shown to be globally convergent to a Mordukhovich-stationary point. Extensive numerical results are given to illustrate the behavior of this method.
\end{abstract}

Key words. cardinality constraints, global minima, local minima, stationary points, M-stationarity, relaxation, regularization method

AMS subject classifications. 90C27, 90C $30,90 \mathrm{C} 46,65 \mathrm{~K} 05$

DOI. $10.1137 / 140978077$

1. Introduction. We consider the cardinality-constrained optimization problem

$$
\min _{x} f(x) \text { s.t. } \quad x \in X,\|x\|_{0} \leq \varkappa,
$$

where $f: \mathbb{R}^{n} \rightarrow \mathbb{R}$ denotes a continuously differentiable function; $\varkappa>0$ is a given natural number; $\|x\|_{0}$ denotes the cardinality of the vector $x \in \mathbb{R}^{n}$, i.e., the number of its nonzero elements; and $X \subseteq \mathbb{R}^{n}$ is a subset determined by any further constraints on $x$. Throughout this manuscript, we assume that $\varkappa<n$, since otherwise the cardinality constraint would not constrain $x$.

The cardinality-constrained optimization problem (1.1) has a wide range of applications including portfolio optimization problems with constraints on the number of assets [6], the subset selection problem in regression [20], and the compressed sensing technique used in signal processing [9]. The optimization problem (1.1) is difficult to solve mainly because it involves the cardinality constraint defined by the mapping $\|\cdot\|_{0}$, which, despite its notation that is quite common in the community, is not a norm and neither convex nor continuous.

The difficulty in solving problem (1.1) is also reflected in the fact that it can be reformulated as a mixed-integer problem. However, even for simple instances, just testing feasibility of the constraints in (1.1) is known to be NP-complete [6].

\footnotetext{
* Received by the editors July 18, 2014; accepted for publication (in revised form) October 20, 2015; published electronically February 4, 2016.

http://www.siam.org/journals/siopt/26-1/97807.html

${ }^{\dagger}$ Department of Mathematics, Linköping University, SE-58183 Linköping, Sweden (oleg. burdakov@liu.se).

${ }^{\ddagger}$ Institute of Mathematics, University of Würzburg, 97074 Würzburg, Germany (kanzow@ mathematik.uni-wuerzburg.de).

$\S$ Graduate School of Computational Engineering, Technical University of Darmstadt, 64293 Darmstadt, Germany (schwartz@gsc.tu-darmstadt.de).
} 
Nevertheless, the mixed-integer formulation of the cardinality-constrained problem is the basis for the development of many algorithms which use ideas and techniques from discrete optimization in order to find the exact or an approximate solution of problem (1.1). We refer the reader to $[5,6,10,21,26,31,32]$ and references therein for a couple of different ideas. Stationarity conditions and algorithms for the unconstrained case $X=\mathbb{R}^{n}$ can be found in [3].

The cardinality-constrained problem (1.1) is also closely related to the sparse optimization problem, where the term $\|x\|_{0}$ is typically a part of the objective function used for enhancing sparsity of produced solutions. A standard technique then is to replace this term by the $l_{1}$-norm $\|x\|_{1}$, which gives rise to a convex optimization problem (provided that all other ingredients are convex) and for which a global minimum can be computed by standard techniques. In general, however, this yields only an approximation of the sparsest solution.

The very recent paper [12] uses a different basic idea and presents a reformulation of the sparse optimization problem as a standard nonlinear program (NLP) with complementarity-type constraints, not involving any integer variables. The so-called "half complementarity" formulation used in that paper corresponds to our reformulation of the cardinality-constrained problem (1.1). Our derivation of this reformulation is different from that used in [12] and provides some insights in itself: We first use another mixed-integer formulation of the cardinality-constrained problem, employing some binary variables, and then show that the standard relaxation of these binary variables, has the nice property that its solutions are still the same as the solutions of the original cardinality-constrained problem (1.1). We presented some preliminary results on this reformulation without proofs in [7]. Apart from this derivation, the remaining part of our paper is, in any case, different from [12]. Nonetheless, some results from the present paper can be translated to sparse optimization problems. A paper discussing the corresponding results and some differences is under preparation.

We should also say that the NLP-reformulation used in [12] and also the one introduced here yield an NLP whose structure is very similar to a mathematical program with complementarity constraints (MPCC); cf. [11, 19, 23]. In fact, it is possible to further rewrite the NLP-reformulation in such a way that one really gets an MPCC (this is the "full complementarity" formulation in [12]). Hence, in principle, one might try to apply the full machinery known from MPCCs. However, it turns out that, besides the usual constraint qualifications, also the MPCC-tailored constraint qualifications are typically violated in this case. Despite this negative observation, we show that our current approach has some stronger properties that are not exhibited in the MPCC-context. We comment on this later within the paper.

The organization is as follows: We begin with some background material in section 2. We then present our NLP-reformulation of the cardinality-constrained optimization problem (1.1) and discuss in detail the relation between the global and local minima in section 3. Stationary conditions of our NLP-reformulation are discussed separately in section 4; here the difficulty is that standard constraint qualifications are usually violated by our NLP-reformulation. Nevertheless, it is shown that the usual KKT-conditions are necessary optimality conditions for the case of a polyhedral convex set $X$, whereas this is not true even if $X$ is convex and satisfies the Slater constraint qualification. The previous discussion motivates us to consider a suitable regularization method for the solution of the cardinality-constrained problem (1.1), which we describe and analyze in section 5 . Extensive numerical results are presented in section 6 , and we conclude with some final remarks in section 7 . 
Notation: The vector $e:=(1, \ldots, 1)^{T} \in \mathbb{R}^{n}$ denotes the all-ones vector, whereas $e_{i}:=(0, \ldots, 0,1,0, \ldots, 0)^{T} \in \mathbb{R}^{n}$ is the $i$ th unit vector. With $B_{r}(a):=\left\{x \mid\|x-a\|_{2} \leq\right.$ $r\}$ we indicate the closed (Euclidean) ball of radius $r>0$ centered in a given point $a \in \mathbb{R}^{n}$. An inequality $x \geq 0$ for some vector $x$ is defined componentwise. Finally, $\operatorname{supp}(x):=\left\{i \mid x_{i} \neq 0\right\}$ denotes the support of a given vector $x$.

2. Preliminaries. In this section, we recall some basic definitions related to standard NLPs that will play some role in our subsequent analysis.

To this end, consider the optimization problem

$$
\begin{array}{cll}
\min f(x) \text { s.t. } & g_{i}(x) \leq 0 \quad \forall i=1, \ldots, m, \\
& h_{i}(x)=0 \quad \forall i=1, \ldots, p
\end{array}
$$

with some continuously differentiable functions $f, g_{i}, h_{i}: \mathbb{R}^{n} \rightarrow \mathbb{R}$.

Definition 2.1. A vector $x^{*} \in \mathbb{R}^{n}$ is called a stationary point of the NLP (2.1) if there exist Lagrange multipliers $\lambda \in \mathbb{R}^{m}$ and $\mu \in \mathbb{R}^{p}$ such that the following KKT (Karush-Kuhn-Tucker) conditions hold:

$$
\begin{aligned}
\nabla_{x} L\left(x^{*}, \lambda, \mu\right) & =0, \\
\lambda_{i} \geq 0, g_{i}\left(x^{*}\right) \leq 0, \lambda_{i} g_{i}\left(x^{*}\right) & =0 \quad \forall i=1, \ldots, m, \\
h_{i}\left(x^{*}\right) & =0 \quad \forall i=1, \ldots, p,
\end{aligned}
$$

where $L(x, \lambda, \mu):=f(x)+\lambda^{T} g(x)+\mu^{T} h(x)$ denotes the Lagrangian of problem (2.1).

Given a local minimum $x^{*}$ of (2.1) such that certain conditions are satisfied at $x^{*}$, it is possible to show that $x^{*}$ is also a stationary point in the sense of Definition 2.1. The conditions required here are called constraint qualifications (CQ). There are a number of different CQs known for NLPs, and we recall some of them in the following discussion. To this end, let $X:=\{x \mid g(x) \leq 0, h(x)=0\}$ be the feasible set of (2.1), and let us introduce some cones that play an important role in the definition of some of these CQs. The set

$$
T_{X}\left(x^{*}\right):=\left\{d \in \mathbb{R}^{n} \mid \exists\left\{x^{k}\right\} \subseteq X \exists\left\{t_{k}\right\} \downarrow 0: x^{k} \rightarrow x^{*} \text { and } d=\lim _{k \rightarrow \infty} \frac{x^{k}-x^{*}}{t_{k}}\right\}
$$

is called the (Bouligand) tangent cone of the set $X$ at the point $x^{*} \in X$. The corresponding linearization cone of $X$ at $x^{*} \in X$ is given by

$$
L_{X}\left(x^{*}\right):=\left\{d \in \mathbb{R}^{n} \mid \nabla g_{i}\left(x^{*}\right)^{T} d \leq 0\left(i: g_{i}\left(x^{*}\right)=0\right), \nabla h_{i}\left(x^{*}\right)^{T} d=0(i=1, \ldots, p)\right\} .
$$

Note that the inclusion $T_{X}\left(x^{*}\right) \subseteq L_{X}\left(x^{*}\right)$ always holds.

Finally, we recall that the polar cone of an arbitrary cone $C \subseteq \mathbb{R}^{n}$ is defined by

$$
C^{*}:=\left\{w \in \mathbb{R}^{n} \mid w^{T} d \leq 0 \forall d \in C\right\} .
$$

Using this notation, we can state some of the more prominent CQs.

Definition 2.2. Let $x^{*}$ be a feasible point of the NLP (2.1). Then we say that $x^{*}$ satisfies the

(a) linear independence CQ (LICQ) if the gradient vectors

$$
\nabla g_{i}\left(x^{*}\right)\left(i: g_{i}\left(x^{*}\right)=0\right), \quad \nabla h_{i}\left(x^{*}\right)(i=1, \ldots, p)
$$

are linearly independent; 
(b) Mangasarian-Fromovitz CQ (MFCQ) if the gradient vectors $\nabla h_{i}\left(x^{*}\right)(i=$ $1, \ldots, p)$ are linearly independent and, in addition, there exists a vector $d \in \mathbb{R}^{n}$ such that $\nabla h_{i}\left(x^{*}\right)^{T} d=0(\forall i=1, \ldots, p)$ and $\nabla g_{i}\left(x^{*}\right)^{T} d<0\left(\forall i: g_{i}\left(x^{*}\right)=0\right)$ hold;

(c) constant rank CQ (CRCQ) if for any subsets $I_{1} \subseteq\left\{i \mid g_{i}\left(x^{*}\right)=0\right\}$ and $I_{2} \subseteq\{1, \ldots, p\}$ such that the gradient vectors

$$
\nabla g_{i}(x)\left(i \in I_{1}\right), \quad \nabla h_{i}(x)\left(i \in I_{2}\right)
$$

are linearly dependent in $x=x^{*}$, they remain linearly dependent for all $x$ in a neighborhood (in $\mathbb{R}^{n}$ ) of $x^{*}$;

(d) constant positive linear dependence condition (CPLD) if for any subsets $I_{1} \subseteq$ $\left\{i \mid g_{i}\left(x^{*}\right)=0\right\}$ and $I_{2} \subseteq\{1, \ldots, p\}$ such that the gradient vectors

$$
\nabla g_{i}(x)\left(i \in I_{1}\right) \quad \text { and } \quad \nabla h_{i}(x)\left(i \in I_{2}\right)
$$

are positive-linear dependent in $x=x^{*}$ (i.e., there exist multipliers $(\alpha, \beta) \neq 0$ with $\alpha \geq 0$ and $\left.\sum_{i=1}^{m} \alpha_{i} \nabla g_{i}\left(x^{*}\right)+\sum_{i=1}^{p} \beta_{i} \nabla h_{i}\left(x^{*}\right)=0\right)$, they are linearly dependent for all $x$ in a neighborhood (in $\mathbb{R}^{n}$ ) of $x^{*}$;

(e) Abadie CQ (ACQ) if $T_{X}\left(x^{*}\right)=L_{X}\left(x^{*}\right)$ holds;

(f) Guignard CQ (GCQ) if $T_{X}\left(x^{*}\right)^{*}=L_{X}\left(x^{*}\right)^{*}$ holds.

The LICQ, MFCQ, ACQ, and GCQ conditions belong to the standard conditions in the optimization community; see, e.g., [2, 22]. Also CRCQ, introduced originally in [17], has found widespread applications; cf. [17] for some examples. Finally, CPLD might be less known; the condition was introduced in [24] and afterwards shown to be a CQ in [1]. The following implications hold:

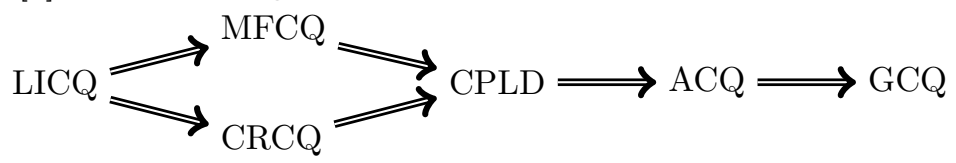

Most of these implications follow immediately from the above definitions. The only nontrivial part is that the ACQ follows from CPLD, a statement that can be derived from $[1,4]$. In view of this diagram, LICQ is the strongest and GCQ the weakest CQ among those given here. In fact, one can show that (in a certain sense) GCQ is the weakest possible CQ which guarantees local minima to be stationary points; see [2].

We close this section with a small example which may be viewed as a special case of the class of problems that will be introduced in the following section and which indicates that GCQ will play a central role in our analysis.

Example 1. Consider the two-dimensional optimization problem

$$
\min _{x, y} f(x) \quad \text { s.t. } \quad x y=0,0 \leq y \leq 1,
$$

where we denote the variables by $x$ and $y$ instead of $x_{1}$ and $x_{2}$ since this simplifies the notation and since this also fits better into the framework that will be discussed later. Geometrically, it is clear (and can also be verified analytically in an easy way) that this simple optimization problem violates ACQ in $\left(x^{*}, y^{*}\right)=(0,0)$, and hence also the stronger conditions LICQ and MFCQ. On the other hand, GCQ is satisfied in $\left(x^{*}, y^{*}\right)$, and thus every local minimum is a stationary point. See Figure 1.

3. Reformulation. This section presents a reformulation of the cardinalityconstrained problem (1.1) as a smooth optimization problem and then discusses the relation between the solutions (in the sense of global minima) and local minima of the original and reformulated problems in sections 3.1 and 3.2, respectively. 


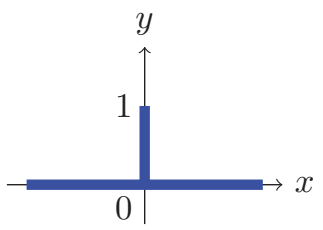

(a) feasible set.

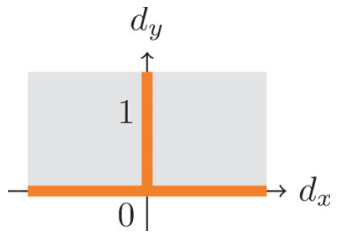

(b) $T_{X}(0,0) \subsetneq L_{X}(0,0)$.

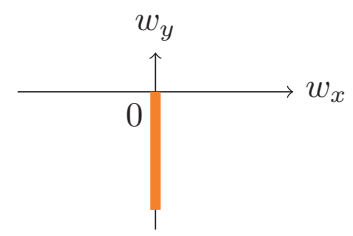

(c) $T_{X}(0,0)^{*}=L_{X}(0,0)^{*}$.

FIG. 1. Illustration of Example 1.

In order to obtain a suitable reformulation of the cardinality-constrained problem (1.1), we first consider the mixed-integer problem

$$
\begin{aligned}
\min _{x, y} f(x) \text { s.t. } & x \in X, \\
& e^{T} y \geq n-\varkappa, \\
& x_{i} y_{i}=0 \quad \forall i=1, \ldots, n, \\
& y_{i} \in\{0,1\} \quad \forall i=1, \ldots, n .
\end{aligned}
$$

Next, we consider the following standard relaxation of (3.1):

$$
\begin{aligned}
\min _{x, y} f(x) \text { s.t. } & x \in X, \\
& e^{T} y \geq n-\varkappa, \\
& x_{i} y_{i}=0 \quad \forall i=1, \ldots, n, \\
& 0 \leq y_{i} \leq 1 \quad \forall i=1, \ldots, n,
\end{aligned}
$$

where the binary constraints are replaced in the usual way by some simple box constraints. The formulation (3.2) will be of central importance for this paper.

Remark 1. Note that the subsequent considerations would also hold with the inequality $e^{T} y \geq n-\varkappa$ in (3.2) being replaced by the equality constraint $e^{T} y=n-\varkappa$. The corresponding modifications are minor. Numerically, we prefer to work with the inequality version because this enlarges the feasible region and therefore provides some more freedom.

3.1. Relation between global minima. According to the next result, the two problems (1.1) and (3.1) have the same solutions in $x$ in the sense of global minima.

THEOREM 3.1. A vector $x^{*} \in \mathbb{R}^{n}$ is a solution of problem (1.1) if and only if there exists a vector $y^{*} \in \mathbb{R}^{n}$ such that $\left(x^{*}, y^{*}\right)$ solves the mixed-integer problem (3.1).

Proof. Since the objective functions of the two problems (1.1) and (3.1) are the same and do not depend on $y$, it suffices to show that $x$ is feasible for (1.1) if and only if there exists a vector $y$ such that $(x, y)$ is feasible for (3.1).

First assume that $x$ is feasible for (1.1). Then, due to $\|x\|_{0} \leq \varkappa$, the vector $y \in \mathbb{R}^{n}$ defined componentwise by

$$
y_{i}:=\left\{\begin{array}{ll}
0 & \text { if } x_{i} \neq 0 \\
1 & \text { if } x_{i}=0
\end{array} \quad \forall i=1, \ldots, n\right.
$$

satisfies $y \in\{0,1\}^{n}, e^{T} y \geq n-\varkappa$, and $x_{i} y_{i}=0$ for all $i=1, \ldots, n$. Hence $(x, y)$ is feasible for problem (3.1).

Conversely, assume that we have a feasible pair $(x, y)$ of problem (3.1). Then define the index set $J:=\left\{i \mid y_{i}=1\right\}$. Since, by assumption, $y_{i} \in\{0,1\}$ and 
$e^{T} y \geq n-\varkappa$, it follows that $|J| \geq n-\varkappa$. Furthermore, using $x_{i} y_{i}=0$ for all $i=1, \ldots, n$, we see that $x_{i}=0$ at least for all $i \in J$; hence $\|x\|_{0} \leq \varkappa$. Thus, $x$ is feasible for problem (1.1).

The following result states that the relaxed problem (3.2) is still equivalent to the original cardinality-constrained problem (1.1) in the sense of global minima.

TheOREM 3.2. A vector $x^{*} \in \mathbb{R}^{n}$ is a solution of problem (1.1) if and only if there exists a vector $y^{*} \in \mathbb{R}^{n}$ such that $\left(x^{*}, y^{*}\right)$ is a solution of the relaxed problem (3.2).

Proof. By analogy with the proof of Theorem 3.1, it can be shown that a vector $x$ is feasible for (1.1) if and only if there exists a vector $y$ such that $(x, y)$ is feasible for (3.2). (Take $J:=\left\{i \mid y_{i} \in(0,1]\right\}$ instead of $J=\left\{i \mid y_{i}=1\right\}$ in the previous proof.) Since the objective function of both problems is the same, this implies the assertion.

An immediate consequence of the previous theorem is the following existence result.

Theorem 3.3. Suppose that the feasible set $\mathcal{F}:=\left\{x \in X \mid\|x\|_{0} \leq \varkappa\right\}$ of the cardinality-constrained problem (1.1) is nonempty and that $X$ is compact. Then both problem (1.1) and the relaxed problem (3.2) have a nonempty solution set.

Proof. First note that the set $\mathcal{C}:=\left\{x \in \mathbb{R}^{n} \mid\|x\|_{0} \leq \varkappa\right\}$ is obviously closed. Hence the feasible set $\mathcal{F}$ of (1.1) is the intersection of a compact set $X$ with a closed set $\mathcal{C}$ and, therefore, compact. Since the objective function $f$ is continuous, it follows that the cardinality-constrained optimization problem (1.1) has a nonempty solution set, and by Theorem 3.2 this implies that the relaxed problem (3.2) is also solvable.

3.2. Relation between local minima. In view of Theorem 3.2, there is a oneto-one correspondence between the solutions of the original problem (1.1) and the solutions of the relaxed problem (3.2). Our next aim is to investigate the relation between the local minima of these two optimization problems. The following result shows that every local minimum of the given cardinality-constrained problem yields a local minimum of the relaxed problem (3.2).

TheOrem 3.4. Let $x^{*} \in \mathbb{R}^{n}$ be a local minimum of (1.1). Then there exists a vector $y^{*} \in \mathbb{R}^{n}$ such that the pair $\left(x^{*}, y^{*}\right)$ is also a local minimum of (3.2).

Proof. Let us define a vector $y^{*}$ componentwise by

$$
y_{i}^{*}:=\left\{\begin{array}{ll}
1 & \text { if } x_{i}^{*}=0 \\
0 & \text { if } x_{i}^{*} \neq 0
\end{array} \quad \forall i=1, \ldots, n\right.
$$

Then we have $y_{i}^{*}=1$ if and only if $x_{i}^{*}=0$ and hence $e^{T} y^{*}=n-\left\|x^{*}\right\|_{0} \geq n-\varkappa$. It is easy to see that $\left(x^{*}, y^{*}\right)$ is feasible for problem (3.2). We claim that $\left(x^{*}, y^{*}\right)$ is a local minimum of (3.2). To this end, first note that there exists an $r_{1}>0$ such that

$$
f(x) \geq f\left(x^{*}\right) \quad \forall x \in X \cap B_{r_{1}}\left(x^{*}\right),\|x\|_{0} \leq \varkappa,
$$

due to the assumed local optimality of $x^{*}$ for problem (1.1). Furthermore, let us choose $r_{2}=\frac{1}{2}$. Then we have $y_{i}>0$ for all $y \in B_{r_{2}}\left(y^{*}\right)$ and all $i$ such that $y_{i}^{*}>0$. This observation immediately yields the inclusion

$$
\left\{i \mid y_{i}=0\right\} \subseteq\left\{i \mid y_{i}^{*}=0\right\} \quad \forall y \in B_{r_{2}}\left(y^{*}\right) .
$$

Now take $r:=\min \left\{r_{1}, r_{2}\right\}$, and let $(x, y) \in B_{r}\left(x^{*}\right) \times B_{r}\left(y^{*}\right)$ be an arbitrary feasible vector of the relaxed problem (3.2). Then, in particular, we have $x \in X$. Moreover, 
the inclusion (3.3) implies

$$
x_{i} \neq 0 \quad \Longrightarrow \quad y_{i}=0 \quad \Longrightarrow \quad y_{i}^{*}=0 \quad \Longrightarrow \quad x_{i}^{*} \neq 0
$$

and therefore shows that $\|x\|_{0} \leq\left\|x^{*}\right\|_{0}$. Hence $x$ is feasible for problem (1.1). Since we also have $x \in B_{r_{1}}\left(x^{*}\right)$, we obtain $f(x) \geq f\left(x^{*}\right)$ from the local optimality of $x^{*}$ for problem (1.1). Consequently, $\left(x^{*}, y^{*}\right)$ is a local minimum of the relaxed problem (3.2).

Note that if $\left\|x^{*}\right\|_{0}=\varkappa$, then the vector $y^{*}$ in Theorem 3.4 is unique; i.e., there exists exactly one $y^{*}$ such that $\left(x^{*}, y^{*}\right)$ is a local minimum of (3.2) (see Proposition 3.5 below). If $\left\|x^{*}\right\|_{0}<\varkappa$, then $y^{*}$ is not unique. Unfortunately, the converse of Theorem 3.4 is not true in general. This is shown by the following counterexample.

Example 2. Consider the three-dimensional problem

$$
\min _{x}\|x-a\|_{2}^{2} \quad \text { s.t. }\|x\|_{0} \leq \varkappa, x \in \mathbb{R}^{3},
$$

with $a:=(1,2,3)^{T}$ and $\varkappa:=2$. This problem has a unique global minimizer at

$$
x^{*}:=(0,2,3)^{T}
$$

as well as two local minimizers at

$$
x^{1}:=(1,0,3)^{T} \quad \text { and } \quad x^{2}:=(1,2,0)^{T} .
$$

On the other hand, the relaxed problem (3.2) has a unique global minimum at

$$
x^{*}:=(0,2,3)^{T}, \quad y^{*}:=(1,0,0)^{T}
$$

(this is consistent with Theorem 3.2), but the number of local minima is larger; namely, they are

$$
\begin{array}{lll}
x^{1}:=(1,0,3)^{T}, & y^{1}:=(0,1,0)^{T}, & \\
x^{2}:=(1,2,0)^{T}, & y^{2}:=(0,0,1)^{T}, & \\
x^{3}:=(1,0,0)^{T}, & y^{3}:=(0, t, 1-t)^{T} & \forall t \in(0,1), \\
x^{4}:=(0,2,0)^{T}, & y^{4}:=(t, 0,1-t)^{T} & \forall t \in(0,1), \\
x^{5}:=(0,0,3)^{T}, & y^{5}:=(t, 1-t, 0)^{T} & \forall t \in(0,1), \\
x^{6}:=(0,0,0)^{T}, & y^{6}:=\left(t_{1}, t_{2}, t_{3}\right)^{T} & \forall t_{i}>0 \text { s.t. } t_{1}+t_{2}+t_{3}=1 .
\end{array}
$$

Note that the corresponding $y^{i}$ is neither unique nor binary for $i=3,4,5,6$, i.e., for all those $x^{i}$ which are not local minima of (1.1).

Let $\left(x^{*}, y^{*}\right)$ be a local minimizer of problem (3.2). One may think that if $y^{*}$ is binary, then $x^{*}$ is a local minimizer of problem (1.1). Unfortunately, this claim is not true in general. We demonstrate this by a modification of the previous counterexample.

Example 3. Consider once again the three-dimensional cardinality-constrained problem from (3.4), but this time with $a:=(1,2,0)^{T}$ and the cardinality number $\varkappa:=$ 1. Here, it is easy to see that the pair $\left(x^{*}, y^{*}\right)$ with $x^{*}:=(0,0,0)^{T}, y^{*}:=(1,1,0)^{T}$ is a local minimizer of the corresponding relaxed problem (3.2) with a binary vector $y^{*}$, while $x^{*}$ is not a local minimizer of (1.1). Note, however, that the vector $y^{*}$ is not unique in this case.

The previous two examples illustrate that the relation between the local minima of the two problems (1.1) and (3.2) is not as straightforward as for the global minima. 
A central observation in this context is that those local minima of the relaxed problem, which are also local minima of the original problem, satisfy the cardinality constraint $\|x\|_{0} \leq \varkappa$ with equality, which, in view of the subsequent result, is equivalent to the statement that the vector $y^{*}$ defined by $x^{*}$ is unique.

Proposition 3.5. Let $\left(x^{*}, y^{*}\right)$ be a local minimum of problem (3.2). Then $\left\|x^{*}\right\|_{0}=\varkappa$ holds if and only if $y^{*}$ is unique, i.e., if there is exactly one $y^{*}$ such that $\left(x^{*}, y^{*}\right)$ is a local minimum of (3.2). In this case, the components of $y^{*}$ are binary.

Proof. First assume that $\left\|x^{*}\right\|_{0}=\varkappa$ holds. Then it follows immediately from the constraints in (3.2) that there exists a unique vector $y^{*}$ such that $\left(x^{*}, y^{*}\right)$ is feasible for problem (3.2). The components of this vector $y^{*}$ are obviously given by

$$
y_{i}^{*}:=\left\{\begin{array}{ll}
1 & \text { if } x_{i}^{*}=0 \\
0 & \text { if } x_{i}^{*} \neq 0
\end{array} \quad \forall i=1, \ldots, n\right.
$$

and thus are binary.

Conversely, suppose that $y^{*}$ is unique. To prove that $\left\|x^{*}\right\|_{0}=\varkappa$, we assume, on the contrary, that $\left\|x^{*}\right\|_{0}<\varkappa$. Since this implies $\left\|x^{*}\right\|_{0} \leq n-2$ (recall that $\varkappa<n$ ), we can find $j_{1} \neq j_{2}$ such that $x_{j_{1}}^{*}=x_{j_{2}}^{*}=0$. Then consider the vectors $y^{\prime}, y^{\prime \prime} \in \mathbb{R}^{n}$ with components defined by

$$
y_{i}^{\prime}:=\left\{\begin{array}{ll}
1 & \text { if } x_{i}^{*}=0, \\
0 & \text { if } x_{i}^{*} \neq 0,
\end{array} \quad y_{i}^{\prime \prime}:= \begin{cases}\frac{1}{2} & \text { if } i \in\left\{j_{1}, j_{2}\right\} \\
1 & \text { if } x_{i}^{*}=0, i \notin\left\{j_{1}, j_{2}\right\}, \quad \forall i=1, \ldots, n . \\
0 & \text { if } x_{i}^{*} \neq 0,\end{cases}\right.
$$

Then obviously $y^{\prime} \neq y^{\prime \prime}$, but $\left(x^{*}, y^{\prime}\right)$ and $\left(x^{*}, y^{\prime \prime}\right)$ are both feasible for (3.2) since, e.g.,

$$
e^{T} y^{\prime \prime}=n-\left\|x^{*}\right\|_{0}-1 \geq n-(\varkappa-1)-1=n-\varkappa .
$$

Similar to the proof of Theorem 3.4, it can be verified that both $\left(x^{*}, y^{\prime}\right)$ and $\left(x^{*}, y^{\prime \prime}\right)$ are local minima of problem (3.2), thus contradicting the uniqueness of $y^{*}$. Hence, we necessarily have $\left\|x^{*}\right\|_{0}=\varkappa$, which, as was noted above, implies that $y^{*}$ is binary.

We are finally in the position to prove a special case of the converse of Theorem 3.4.

TheOrem 3.6. Let $\left(x^{*}, y^{*}\right)$ be a local minimizer of problem (3.2) with $\left\|x^{*}\right\|_{0}=\varkappa$. Then $x^{*}$ is a local minimum of the cardinality-constrained problem (1.1).

Proof. By assumption, there exists some number $r_{1}>0$ such that $\left(x^{*}, y^{*}\right)$ is a minimum of the relaxed problem (3.2) in a neighborhood $B_{r_{1}}\left(x^{*}\right) \times B_{r_{1}}\left(y^{*}\right)$ of $\left(x^{*}, y^{*}\right)$. Let us choose

$$
r_{2}>0 \quad \text { with } \quad r_{2}<\min \left\{\left|x_{i}^{*}\right| \mid x_{i}^{*} \neq 0\right\}
$$

and $r:=\min \left\{r_{1}, r_{2}\right\}$. We claim that $x^{*}$ is a minimum of the cardinality-constrained problem (1.1) in the neighborhood $B_{r}\left(x^{*}\right)$. To this end, let $x \in B_{r}\left(x^{*}\right)$ be an arbitrary feasible point of problem (1.1). By definition of $r_{2}$ and $r$, we have

$$
x_{i}^{*} \neq 0 \quad \Longrightarrow \quad x_{i} \neq 0 \quad \forall i=1, \ldots, n,
$$

which implies $\varkappa=\left\|x^{*}\right\|_{0} \leq\|x\|_{0}$. By the feasibility of $x$ we know $\|x\|_{0} \leq \varkappa$ and thus

$$
\left\{i \mid x_{i}^{*} \neq 0\right\}=\left\{i \mid x_{i} \neq 0\right\},
$$

or, equivalently, that

$$
\left\{i \mid x_{i}^{*}=0\right\}=\left\{i \mid x_{i}=0\right\}
$$

Copyright $@$ by SIAM. Unauthorized reproduction of this article is prohibited. 
This, however, implies that $\left(x, y^{*}\right)$ is also feasible for the relaxed problem (3.2) satisfying $\left(x, y^{*}\right) \in B_{r}\left(x^{*}\right) \times B_{r}\left(y^{*}\right)$. Consequently, we obtain $f(x) \geq f\left(x^{*}\right)$ from the local optimality of $\left(x^{*}, y^{*}\right)$ for problem (3.2). Altogether, this shows that $x^{*}$ is a local minimum of (1.1).

Regarding the additional assumption $\left\|x^{*}\right\|_{0}=\varkappa$ used in Theorem 3.6: Of course it depends on the concrete problem whether this condition is satisfied in a global minimum of (1.1). However, whenever the cardinality constraint is a critical resource constraint, it is not unreasonable to assume that it is active in a global solution.

We close this section with a short comparison of our reformulation with the more standard one used in [6].

Remark 2. Consider the cardinality-constrained optimization problem (1.1), and assume, in addition, that the set $X$ includes lower and upper bounds on the variables $x_{i}$, say $0 \leq x_{i} \leq u_{i}$ for all $i=1, \ldots, n$. Then, suppressing all other constraints, our complementarity-type reformulation yields the equivalence

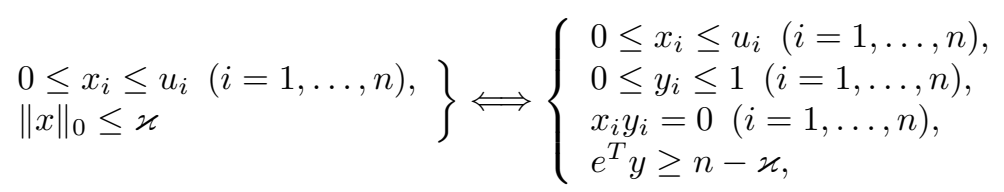

whereas the mixed-integer program suggested in [6] provides the equivalence

$$
\left.\begin{array}{l}
0 \leq x_{i} \leq u_{i}(i=1, \ldots, n), \\
\|x\|_{0} \leq \varkappa
\end{array}\right\} \Longleftrightarrow\left\{\begin{array}{l}
0 \leq x_{i} \leq u_{i}\left(1-y_{i}\right)(i=1, \ldots, n), \\
y_{i} \in\{0,1\}(i=1, \ldots, n), \\
e^{T} y \geq n-\varkappa,
\end{array}\right.
$$

whose standard relaxation gives the constraints

$$
0 \leq x_{i} \leq u_{i}\left(1-y_{i}\right), \quad 0 \leq y_{i} \leq 1 \quad(i=1, \ldots, n), \quad e^{T} y \geq n-\varkappa
$$

which are linear in $x$ and $y$ but no longer equivalent to the cardinality constraints. It is interesting to compare this formulation with our complementarity-type reformulation. To this end, we neglect the constraint $e^{T} y \geq n-\varkappa$, which is used in both cases, and consider a single component $i$ of the vectors $x_{i}$ and $y_{i}$. Then we have the constraints

$$
0 \leq x_{i} \leq u_{i}, \quad 0 \leq y_{i} \leq 1, \quad x_{i} y_{i}=0,
$$

whereas [6] yields

$$
0 \leq x_{i} \leq u_{i}\left(1-y_{i}\right), \quad 0 \leq y_{i} \leq 1
$$

The sets described by (3.5) and (3.6) are shown in Figures 2(a) and (b), respectively. It follows that (3.6) is simply the convex hull of our reformulation (3.5). Apart from this relation, we note, however, that our formulation can also be used when there are no lower or upper bounds on the variables.

4. Stationarity conditions. Here we investigate the question whether the standard KKT conditions are necessary optimality conditions for the relaxed program (3.2) or whether we have to deal with a weaker stationary concept in general. It turns out that the KKT conditions are indeed satisfied for the case where $X$ is polyhedral convex, whereas this is no longer true (in general) for the case of a nonlinear set $X$. We therefore divide this section into two subsections, 4.1 and 4.2 , where we discuss the linear and the nonlinear cases separately. 


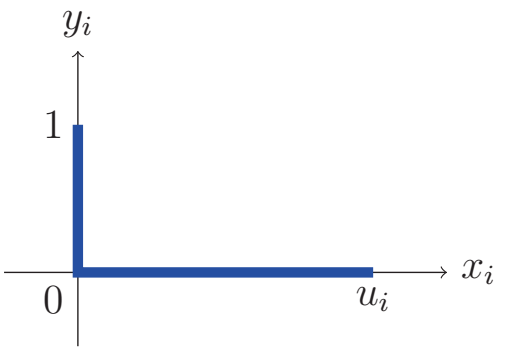

(a) $0 \leq x_{i} \leq u_{i}, \quad 0 \leq y_{i} \leq 1, x_{i} y_{i}=0$.

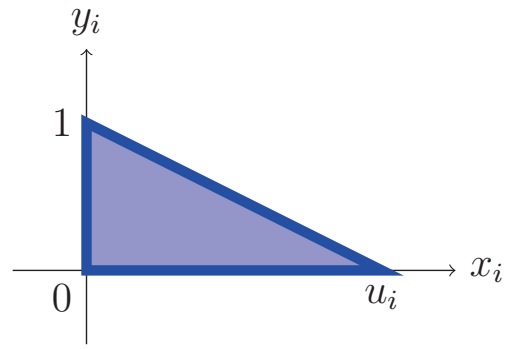

(b) $0 \leq x_{i} \leq u_{i}\left(1-y_{i}\right), 0 \leq y_{i} \leq 1$.

FIG. 2. Comparison of the two different reformulations/relaxations.

4.1. Linear constraints. In order to be able to prove the existence of Lagrange multipliers in a minimum of the reformulated problem (3.2), we consider the special case where $X$ is polyhedral convex, i.e.,

$$
X=\left\{x \in \mathbb{R}^{n} \mid a_{i}^{T} x \leq \alpha_{i}(i=1, \ldots, m), b_{i}^{T} x=\beta_{i}(i=1, \ldots, p)\right\} .
$$

We will show that in this case the GCQ is satisfied in every feasible point, and thus every local minimum of (3.2) is a KKT point.

To this end, let us denote the feasible set of (3.2) by $Z$, and define the following index sets for all $\left(x^{*}, y^{*}\right) \in Z$ :

$$
\begin{aligned}
I_{a}\left(x^{*}\right) & :=\left\{i \in\{1, \ldots, m\} \mid a_{i}^{T} x^{*}=\alpha_{i}\right\}, \\
I_{0}\left(x^{*}\right) & :=\left\{i \in\{1, \ldots, n\} \mid x_{i}^{*}=0\right\}, \\
I_{ \pm 0}\left(x^{*}, y^{*}\right) & :=\left\{i \in\{1, \ldots, n\} \mid x_{i}^{*} \neq 0, y_{i}^{*}=0\right\}, \\
I_{00}\left(x^{*}, y^{*}\right) & :=\left\{i \in\{1, \ldots, n\} \mid x_{i}^{*}=0, y_{i}^{*}=0\right\}, \\
I_{0+}\left(x^{*}, y^{*}\right) & :=\left\{i \in\{1, \ldots, n\} \mid x_{i}^{*}=0, y_{i}^{*} \in(0,1)\right\}, \\
I_{01}\left(x^{*}, y^{*}\right) & :=\left\{i \in\{1, \ldots, n\} \mid x_{i}^{*}=0, y_{i}^{*}=1\right\} .
\end{aligned}
$$

Note that the two index sets $I_{0}\left(x^{*}\right)$ and $I_{ \pm 0}\left(x^{*}, y^{*}\right)$ form a partition of the set $\{1, \ldots, n\}$, whereas $I_{0}\left(x^{*}\right)$ itself gets partitioned into the three subsets $I_{00}\left(x^{*}, y^{*}\right)$, $I_{0+}\left(x^{*}, y^{*}\right)$, and $I_{01}\left(x^{*}, y^{*}\right)$.

For all subsets $I \subseteq I_{00}\left(x^{*}, y^{*}\right)$ we define the restricted feasible sets

$$
\begin{array}{lll}
Z_{I}:=\left\{(x, y) \in \mathbb{R}^{n} \times \mathbb{R}^{n} \mid\right. & \forall_{i=1, \ldots, m} & a_{i}^{T} x \leq \alpha_{i}, \\
& \forall_{i=1, \ldots, p} & b_{i}^{T} x=\beta_{i}, \\
& & e^{T} y \geq n-\varkappa, \\
& \forall_{i \in I_{0+}\left(x^{*}, y^{*}\right) \cup I_{01}\left(x^{*}, y^{*}\right) \cup I} & x_{i}=0, y_{i} \in[0,1], \\
& \forall_{i \in I_{ \pm 0}\left(x^{*}, y^{*}\right) \cup\left(I_{00}\left(x^{*}, y^{*}\right) \backslash I\right)} & \left.y_{i}=0\right\} .
\end{array}
$$

Then we can rewrite the set $Z$ locally around a feasible point $\left(x^{*}, y^{*}\right)$ as follows.

Proposition 4.1. Let $\left(x^{*}, y^{*}\right) \in Z$ and the sets $Z_{I}$ for $I \subseteq I_{00}\left(x^{*}, y^{*}\right)$ be defined in (4.1). Then the following statements hold:

(a) $\left(x^{*}, y^{*}\right) \in Z_{I}$ for all $I \subseteq I_{00}\left(x^{*}, y^{*}\right)$.

(b) For all $r>0$ sufficiently small

$$
Z \cap B_{r}\left(x^{*}, y^{*}\right)=\left(\bigcup_{I \subseteq I_{00}\left(x^{*}, y^{*}\right)} Z_{I}\right) \cap B_{r}\left(x^{*}, y^{*}\right) .
$$

Copyright (c) by SIAM. Unauthorized reproduction of this article is prohibited. 
Proof. Statement (a) follows directly from the definition of the sets $Z_{I}$. Hence we have to prove only (b). By definition $Z_{I} \subseteq Z$ for all $I \subseteq I_{00}\left(x^{*}, y^{*}\right)$. This implies

$$
Z \cap B_{r}\left(x^{*}, y^{*}\right) \supseteq\left(\bigcup_{I \subseteq I_{00}\left(x^{*}, y^{*}\right)} Z_{I}\right) \cap B_{r}\left(x^{*}, y^{*}\right) .
$$

Now consider an arbitrary element $(x, y) \in Z \cap B_{r}\left(x^{*}, y^{*}\right)$. Then $x \in X$ and $e^{T} y \geq$ $n-\varkappa$. For all $r>0$ sufficiently small, $i \in I_{0+}\left(x^{*}, y^{*}\right) \cup I_{01}\left(x^{*}, y^{*}\right)$ implies $y_{i} \in(0,1]$ and thus $x_{i}=0$. Analogously, we get $x_{i} \neq 0$ and thus $y_{i}=0$ for all $i \in I_{ \pm 0}\left(x^{*}, y^{*}\right)$. Now define $I:=\left\{i \in I_{00}\left(x^{*}, y^{*}\right) \mid x_{i}=0\right\}$. Due to the feasibility of $(x, y)$, this implies $y_{i} \in[0,1]$ for all $i \in I$ and $y_{i}=0$ for all $i \in I_{00}\left(x^{*}, y^{*}\right) \backslash I$. Thus, we have proven $(x, y) \in Z_{I}$, and consequently the opposite inclusion holds as well.

This result can be used to replace the tangent cone $T_{Z}\left(x^{*}, y^{*}\right)$ and its polar cone $T_{Z}\left(x^{*}, y^{*}\right)^{*}$ by unions and intersections of simpler cones.

LEMmA 4.2. Let $\left(x^{*}, y^{*}\right) \in Z$ and the sets $Z_{I}$ for $I \subseteq I_{00}\left(x^{*}, y^{*}\right)$ be defined in (4.1). Then the tangent cone and its polar satisfy the following equations:

(a) $T_{Z}\left(x^{*}, y^{*}\right)=\bigcup_{I \subseteq I_{00}\left(x^{*}, y^{*}\right)} T_{Z_{I}}\left(x^{*}, y^{*}\right)$.

(b) $T_{Z}\left(x^{*}, y^{*}\right)^{*}=\bigcap_{I \subseteq I_{00}\left(x^{*}, y^{*}\right)} T_{Z_{I}}\left(x^{*}, y^{*}\right)^{*}$.

Proof. Let $r>0$ be sufficiently small such that Proposition 4.1 holds. Then statement (a) follows from

$$
\begin{aligned}
T_{Z}\left(x^{*}, y^{*}\right) & =T_{Z \cap B_{r}\left(x^{*}, y^{*}\right)}\left(x^{*}, y^{*}\right) \\
& =T_{\left(\bigcup_{I \subseteq I_{00}\left(x^{*}, y^{*}\right)} Z_{I}\right) \cap B_{r}\left(x^{*}, y^{*}\right)}\left(x^{*}, y^{*}\right) \\
& =T_{\bigcup_{I \subseteq I_{00}\left(x^{*}, y^{*}\right)} Z_{I}}\left(x^{*}, y^{*}\right) \\
& =\bigcup_{I \subseteq I_{00}\left(x^{*}, y^{*}\right)} T_{Z_{I}}\left(x^{*}, y^{*}\right),
\end{aligned}
$$

where the first and third equations follow from the fact that the tangent cone, by definition, depends only on the local properties around $\left(x^{*}, y^{*}\right)$; the second equality comes from Proposition 4.1; while the final identity is again a direct consequence of the definition of the tangent cone, taking into account that we have the union of only finitely many sets here. Statement (b) is then a direct application of [2, Theorem $3.1 .9]$ to the nonempty cones $T_{Z_{I}}\left(x^{*}, y^{*}\right)$.

To verify GCQ, we now have to calculate the polar cones $T_{Z_{I}}\left(x^{*}, y^{*}\right)^{*}$ and their intersection $T_{Z}\left(x^{*}, y^{*}\right)^{*}$. However, since the sets $Z_{I}$ are polyhedral convex, calculating the polar cones $T_{Z_{I}}\left(x^{*}, y^{*}\right)^{*}$ is straightforward.

Lemma 4.3. Let $\left(x^{*}, y^{*}\right) \in Z$ and the sets $Z_{I}$ for $I \subseteq I_{00}\left(x^{*}, y^{*}\right)$ as in (4.1).

(a) For all $I \subseteq I_{00}\left(x^{*}, y^{*}\right)$ we have

$$
\begin{aligned}
& T_{Z_{I}}\left(x^{*}, y^{*}\right)^{*}=\left\{\left(w_{x}, w_{y}\right) \in \mathbb{R}^{n} \times \mathbb{R}^{n} \mid w_{x}=\sum_{i \in I_{a}\left(x^{*}\right)} \lambda_{i} a_{i}+\sum_{i=1}^{p} \mu_{i} b_{i}+\sum_{i=1}^{n} \gamma_{i} e_{i},\right. \\
& w_{y}=\delta e+\sum_{i=1}^{n} \nu_{i} e_{i}, \\
& \forall_{i \in I_{a}\left(x^{*}\right)} \quad \lambda_{i} \geq 0 \\
& \delta \leq 0 \text { and } \delta=0 \quad \text { if } e^{T} y^{*}>n-\varkappa \text {, } \\
& \forall_{i \in I_{0+}\left(x^{*}, y^{*}\right)} \nu_{i}=0 \\
& \forall i \in I \quad \nu_{i} \leq 0, \\
& \forall_{i \in I_{01}\left(x^{*}, y^{*}\right)} \quad \nu_{i} \geq 0,
\end{aligned}
$$

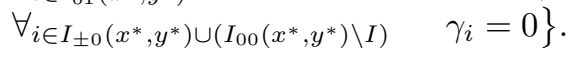

Copyright $@$ by SIAM. Unauthorized reproduction of this article is prohibited. 
(b) The polar cone $T_{Z}\left(x^{*}, y^{*}\right)^{*}$ is given by

$$
\begin{aligned}
T_{Z}\left(x^{*}, y^{*}\right)^{*}=\left\{\left(w_{x}, w_{y}\right) \in \mathbb{R}^{n} \times \mathbb{R}^{n} \mid\right. & w_{x}=\sum_{i \in I_{a}\left(x^{*}\right)} \lambda_{i} a_{i}+\sum_{i=1}^{p} \mu_{i} b_{i}+\sum_{i=1}^{n} \gamma_{i} e_{i}, \\
& w_{y}=\delta e+\sum_{i=1}^{n} \nu_{i} e_{i}, \\
& \forall_{i \in I_{a}\left(x^{*}\right)} \quad \lambda_{i} \geq 0, \\
& \delta \leq 0 \text { and } \delta=0 \quad \text { if } e^{T} y^{*}>n-\varkappa, \\
& \forall_{i \in I_{0+}\left(x^{*}, y^{*}\right)} \quad \nu_{i}=0, \\
& \forall_{i \in I_{00}\left(x^{*}, y^{*}\right)} \quad \gamma_{i}=0, \nu_{i} \leq 0, \\
& \forall_{i \in I_{01}\left(x^{*}, y^{*}\right)} \quad \nu_{i} \geq 0, \\
& \left.\forall_{i \in I_{ \pm 0}\left(x^{*}, y^{*}\right)} \quad \gamma_{i}=0\right\} .
\end{aligned}
$$

Proof. (a) The set $Z_{I}$ is polyhedral convex for all index sets $I \subseteq I_{00}\left(x^{*}, y^{*}\right)$ and can be written as

$$
\begin{array}{lll}
Z_{I}=\left\{(x, y) \in \mathbb{R}^{n} \times \mathbb{R}^{n} \mid\right. & \forall_{i=1, \ldots, m} & \left(a_{i}, 0\right)^{T}(x, y) \leq \alpha_{i}, \\
& \forall_{i=1, \ldots, p} & \left(b_{i}, 0\right)^{T}(x, y)=\beta_{i}, \\
& (0, e)^{T}(x, y) \geq n-x, & \left(e_{i}, 0\right)^{T}(x, y)=0, \\
& \forall_{i \in I_{0+}\left(x^{*}, y^{*}\right) \cup I_{01}\left(x^{*}, y^{*}\right) \cup I} & \left(0, e_{i}\right)^{T}(x, y) \geq 0, \\
& \forall_{i \in I_{0+}\left(x^{*}, y^{*}\right) \cup I_{01}\left(x^{*}, y^{*}\right) \cup I} & \left(0, e_{i}\right)^{T}(x, y) \leq 1, \\
& \forall_{i \in I_{0+}\left(x^{*}, y^{*}\right) \cup I_{01}\left(x^{*}, y^{*}\right) \cup I} & \left.\left(0, e_{i}\right)^{T}(x, y)=0\right\} .
\end{array}
$$

The polar cone $T_{Z_{I}}\left(x^{*}, y^{*}\right)^{*}\left(=N_{Z_{I}}\left(x^{*}, y^{*}\right)\right)$ can thus be calculated using, e.g., [25, Theorem 6.46], which, after some simplification, leads to the formula stated here.

(b) Let us denote the set on the right-hand side of the equation by $W$. By Lemma 4.2, we know $T_{Z}\left(x^{*}, y^{*}\right)^{*}=\bigcap_{I \subseteq I_{00}\left(x^{*}, y^{*}\right)} T_{Z_{I}}\left(x^{*}, y^{*}\right)^{*}$. Since $W \subseteq T_{Z_{I}}\left(x^{*}, y^{*}\right)^{*}$ for all $I \subseteq I_{00}\left(x^{*}, y^{*}\right)$, this implies $W \subseteq T_{Z}\left(x^{*}, y^{*}\right)^{*}$. Now consider an arbitrary element $\left(w_{x}, w_{y}\right) \in T_{Z}\left(x^{*}, y^{*}\right)^{*}$. Choosing $I=\emptyset$, we can conclude $\left(w_{x}, w_{y}\right) \in T_{Z_{\emptyset}}\left(x^{*}, y^{*}\right)^{*}$. Consequently, $w_{x}$ can be written as $w_{x}=\sum_{i \in I_{a}\left(x^{*}\right)} \lambda_{i} a_{i}+\sum_{i=1}^{p} \mu_{i} b_{i}+\sum_{i=1}^{n} \gamma_{i} e_{i}$ with $\lambda_{i} \geq 0$ for all $i \in I_{a}\left(x^{*}\right)$ and $\gamma_{i}=0$ for all $i \in I_{ \pm 0}\left(x^{*}, y^{*}\right) \cup I_{00}\left(x^{*}, y^{*}\right)$. If instead we choose $I=I_{00}\left(x^{*}, y^{*}\right)$, we can write $w_{y}$ as $w_{y}=\delta e+\sum_{i=1}^{n} \nu_{i} e_{i}$ with $\delta \leq 0$ and $\delta=0$ if $e^{T} y^{*}>n-\varkappa, \nu_{i}=0$ for all $i \in I_{0+}\left(x^{*}, y^{*}\right), \nu_{i} \leq 0$ for all $i \in I_{00}\left(x^{*}, y^{*}\right)$, and $\nu_{i} \geq 0$ for all $i \in I_{01}\left(x^{*}, y^{*}\right)$. Consequently, $\left(w_{x}, w_{y}\right) \in W$. Since $\left(w_{x}, w_{y}\right) \in T_{Z}\left(x^{*}, y^{*}\right)^{*}$ was chosen arbitrarily, this implies the missing inclusion.

Note that statement (b) is true only because there are no restrictions in $Z_{I}$ depending on $x$ and $y$ at the same time.

Now it remains to calculate the linearization cone $L_{Z}\left(x^{*}, y^{*}\right)$ and the corresponding polar cone $L_{Z}\left(x^{*}, y^{*}\right)^{*}$.

Lemma 4.4. Let $\left(x^{*}, y^{*}\right) \in Z$ be arbitrarily given. Then the polar cone of $L_{Z}\left(x^{*}, y^{*}\right)$ is given by

$$
\begin{aligned}
L_{Z}\left(x^{*}, y^{*}\right)^{*}=\left\{\left(w_{x}, w_{y}\right) \in \mathbb{R}^{n} \times \mathbb{R}^{n} \mid\right. & w_{x}=\sum_{i \in I_{a}\left(x^{*}\right)} \lambda_{i} a_{i}+\sum_{i=1}^{p} \mu_{i} b_{i}+\sum_{i=1}^{n} \gamma_{i} e_{i}, \\
& w_{y}=\delta e+\sum_{i=1}^{n} \nu_{i} e_{i}, \\
& \forall_{i \in I_{a}\left(x^{*}\right)} \quad \lambda_{i} \geq 0, \\
& \delta \leq 0 \text { and } \delta=0 \quad \text { if } e^{T} y^{*}>n-\varkappa, \\
& \forall_{i \in I_{0+}\left(x^{*}, y^{*}\right)} \quad \nu_{i}=0, \\
& \forall_{i \in I_{00}\left(x^{*}, y^{*}\right)} \quad \gamma_{i}=0, \nu_{i} \leq 0, \\
& \forall_{i \in I_{01}\left(x^{*}, y^{*}\right)} \quad \nu_{i} \geq 0, \\
& \left.\forall_{i \in I_{ \pm 0}\left(x^{*}, y^{*}\right)} \quad \gamma_{i}=0\right\} .
\end{aligned}
$$

Copyright $@$ ( ) by SIAM. Unauthorized reproduction of this article is prohibited. 
Proof. By the definition of the linearization cone, we get

$$
\begin{array}{lll}
L_{Z}\left(x^{*}, y^{*}\right)=\left\{\left(d_{x}, d_{y}\right) \in \mathbb{R}^{n} \times \mathbb{R}^{n} \mid\right. & \forall_{i \in I_{a}\left(x^{*}\right)} & a_{i}^{T} d_{x} \leq 0, \\
& \forall_{i=1, \ldots, p} & b_{i}^{T} d_{x}=0, \\
& e^{T} d_{y} \geq 0 \text { if } e^{T} y^{*}=n-\varkappa, \\
& \forall_{i \in I_{0+}\left(x^{*}, y^{*}\right)}\left(d_{x}\right)_{i}=0, \\
& \forall_{i \in I_{00}\left(x^{*}, y^{*}\right)}\left(d_{y}\right)_{i} \geq 0 \\
& \forall_{i \in I_{01}\left(x^{*}, y^{*}\right)}\left(d_{x}\right)_{i}=0,\left(d_{y}\right)_{i} \leq 0, \\
& \left.\forall_{i \in I_{ \pm 0}\left(x^{*}, y^{*}\right)}\left(d_{y}\right)_{i}=0\right\} .
\end{array}
$$

Since $L_{Z}\left(x^{*}, y^{*}\right)$ is polyhedral convex, the corresponding polar cone can again be calculated using [25, Theorem 6.46], which leads to the given representation.

Using Lemmas 4.3 and 4.4 , we immediately see $T_{Z}\left(x^{*}, y^{*}\right)^{*}=L_{Z}\left(x^{*}, y^{*}\right)^{*}$; i.e., GCQ is satisfied in any feasible point $\left(x^{*}, y^{*}\right) \in Z$, and thus local minima of the reformulated problem (3.2) are KKT points.

COROLlary 4.5. Let $\left(x^{*}, y^{*}\right) \in Z$ be an arbitrary feasible point of (3.2). Then $G C Q$ holds in $\left(x^{*}, y^{*}\right)$.

Note that Example 1 essentially implies that we cannot expect stronger CQs (like the LICQ, MFCQ, or ACQ) to hold.

We also want to stress that Corollary 4.5 points out a significant difference between our class of problems and the closely related mathematical programs with complementarity constraints (MPCC), which are optimization problems of the form

$$
\begin{array}{ll}
\min _{z} f(z) \quad \text { s.t. } & g_{i}(z) \leq 0 \quad \forall i=1, \ldots, m, \\
& h_{i}(z)=0 \quad \forall i=1, \ldots, p, \\
& G_{i}(z) \geq 0, H_{i}(z) \geq 0, G_{i}(z) H_{i}(z)=0 \quad \forall i=1, \ldots, n,
\end{array}
$$

with continuously differentiable functions $f, g_{i}, h_{i}, G_{i}, H_{i}: \mathbb{R}^{n} \rightarrow \mathbb{R}$. If, for example, the set $X$ from (1.1) is given, without loss of generality, in the standard form $X=$ $\{x \mid A x=b, x \geq 0\}$, then our relaxed problem (3.2) is a special case of an MPCC. However, a counterexample in Scheel and Scholtes [27] shows that GCQ may not hold for MPCCs although all functions $g_{i}, h_{i}, G_{i}, H_{i}$ are linear. The reason that we are able to prove the satisfaction of GCQ has to do with the very special structure of our relaxed program, where the two classes of variables $x$ and $y$ are combined only by the complementarity-type constraint, whereas there are no other joint constraints; cf. also the comment after the proof of Lemma 4.3.

4.2. Nonlinear constraints. Here we consider the case where the set $X$ is not (necessarily) polyhedral convex, i.e.,

$$
X=\left\{x \in \mathbb{R}^{n} \mid g_{i}(x) \leq 0(i=1, \ldots, m), h_{i}(x)=0(i=1, \ldots, p)\right\}
$$

with continuously differentiable functions $g_{i}, h_{i}: \mathbb{R}^{n} \rightarrow \mathbb{R}$. In the subsequent discussion, we use the same index sets as in the linear case with the exception of $I_{a}\left(x^{*}\right)$, which is replaced by

$$
I_{g}\left(x^{*}\right)=\left\{i \in\{1, \ldots, m\} \mid g_{i}\left(x^{*}\right)=0\right\} .
$$

The nonlinear case is much more delicate since it turns out that GCQ may not be satisfied. This is illustrated by the following example.

Example 4. Consider the convex, but not polyhedral convex, set

$$
X:=\left\{x \in \mathbb{R}^{2} \mid x_{1}^{2}+\left(x_{2}-1\right)^{2} \leq 1\right\}
$$

Copyright (c) by SIAM. Unauthorized reproduction of this article is prohibited. 
and $f(x)=x_{1}+x_{2}^{2}$. See Figure 3 . When we choose $\varkappa=1$, the unique global solution of the cardinality-constrained problem (1.1) is $x^{*}=(0,0)$. Since $\left\|x^{*}\right\|_{0}=0<\varkappa$, the corresponding $y^{*}$ is not uniquely determined. If we choose $y^{*}=(0,1)$, then $\left(x^{*}, y^{*}\right)$ is a global solution of the relaxed problem (3.2). However, one easily verifies that it is not a KKT point of (3.2), and thus GCQ cannot be satisfied in $\left(x^{*}, y^{*}\right)$.

Note that other pairs such as $\left(x^{*}, \tilde{y}\right)$ with $\tilde{y}=(1,1)$ are KKT points of $(3.2)$.

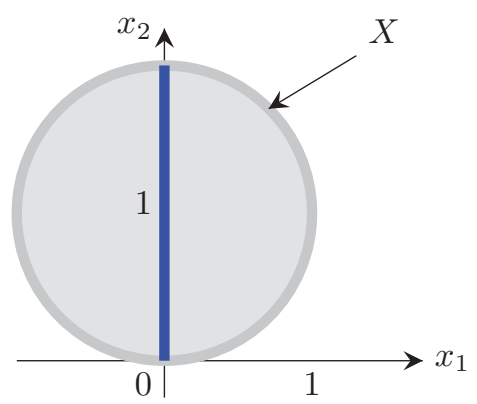

FIG. 3. Illustration of Example 4.

The previous example shows that, for nonlinear sets $X$ (even if $X$ is convex and satisfies the Slater condition), we have to deal with another stationary concept than the usual KKT conditions. This more suitable stationary concept is the M-stationary part of the subsequent definition.

Definition 4.6. Let $\left(x^{*}, y^{*}\right)$ be feasible for the relaxed program (3.2). Then $\left(x^{*}, y^{*}\right)$ is called the following:

(a) S-stationary ( $S=$ strong) if there exist multipliers $\lambda \in \mathbb{R}^{m}, \mu \in \mathbb{R}^{p}$, and $\gamma \in \mathbb{R}^{n}$ such that the following conditions hold:

$$
\begin{array}{r}
\nabla f\left(x^{*}\right)+\sum_{i=1}^{m} \lambda_{i} \nabla g_{i}\left(x^{*}\right)+\sum_{i=1}^{p} \mu_{i} \nabla h_{i}\left(x^{*}\right)+\sum_{i=1}^{n} \gamma_{i} e_{i}=0 \\
\lambda_{i} \geq 0, \quad \lambda_{i} g_{i}\left(x^{*}\right)=0 \quad \forall i=1, \ldots, m \\
\gamma_{i}=0 \quad \forall i \text { s.t. } y_{i}^{*}=0 .
\end{array}
$$

(b) M-stationary ( $M=$ Mordukhovich) if there exist multipliers $\lambda \in \mathbb{R}^{m}, \mu \in \mathbb{R}^{p}$, and $\gamma \in \mathbb{R}^{n}$ such that the following conditions hold:

$$
\begin{aligned}
\nabla f\left(x^{*}\right)+\sum_{i=1}^{m} \lambda_{i} \nabla g_{i}\left(x^{*}\right)+\sum_{i=1}^{p} \mu_{i} \nabla h_{i}\left(x^{*}\right)+\sum_{i=1}^{n} \gamma_{i} e_{i}=0, \\
\lambda_{i} \geq 0, \lambda_{i} g_{i}\left(x^{*}\right)=0 \quad \forall i=1, \ldots, m \\
\gamma_{i}=0 \quad \forall i \text { s.t. } x_{i}^{*} \neq 0 .
\end{aligned}
$$

The terminology used in the previous definition is similar to the one in the MPECsetting. Note that the only difference in the two definitions is that S-stationarity requires $\gamma_{i}=0$ for all indices $i$ such that $y_{i}^{*}=0$, whereas M-stationarity says that this has to hold only for those indices $i$ where $x_{i}^{*} \neq 0$ (recall that the feasibility of $\left(x^{*}, y^{*}\right)$ then implies $\left.y_{i}^{*}=0\right)$, but M-stationarity does not require anything for the multipliers $\gamma_{i}$ for the biactive indices where we have $x_{i}^{*}=0$ and $y_{i}^{*}=0$; hence M-stationarity is a weaker condition than S-stationarity. 
Of course, the definitions of S- and M-stationarity are completely unmotivated so far. As for S-stationarity, the following result simply says that this is just a reformulation of the standard KKT conditions.

Proposition 4.7. Let $\left(x^{*}, y^{*}\right)$ be feasible for the relaxed program (3.2) with $X$ defined by (4.2). Then $\left(x^{*}, y^{*}\right)$ is a stationary point of (3.2), i.e., satisfies the usual $K K T$ conditions, if and only if $\left(x^{*}, y^{*}\right)$ is an S-stationary point.

Proof. Let $\left(x^{*}, y^{*}\right)$ be a stationary point of (3.2). Then there exist Lagrange multipliers $\lambda, \mu, \rho, \tilde{\gamma}, \nu^{+}, \nu^{-}$such that the following KKT conditions hold:

$$
\begin{aligned}
\nabla f\left(x^{*}\right)+\sum_{i=1}^{m} \lambda_{i} \nabla g_{i}\left(x^{*}\right)+\sum_{j=1}^{p} \mu_{j} \nabla h_{j}\left(x^{*}\right)+\sum_{i=1}^{n} \tilde{\gamma}_{i} y_{i}^{*} e_{i}=0 \\
-\delta e+\sum_{i=1}^{n} \tilde{\gamma}_{i} x_{i}^{*} e_{i}+\sum_{i=1}^{n}\left(\nu_{i}^{+}-\nu_{i}^{-}\right) e_{i}=0 \\
\lambda_{i} \geq 0, \quad \lambda_{i} g_{i}\left(x^{*}\right)=0 \quad \forall i=1, \ldots, m, \\
\delta \geq 0, \delta\left(e^{T} y^{*}-n+\varkappa\right)=0 \\
\nu_{i}^{+} \geq 0, \nu_{i}^{+}\left(y_{i}^{*}-1\right)=0 \quad \forall i=1, \ldots, n, \\
\nu_{i}^{-} \geq 0, \nu_{i}^{-} y_{i}^{*}=0 \quad \forall i=1, \ldots, n .
\end{aligned}
$$

Setting $\gamma_{i}:=\tilde{\gamma}_{i} y_{i}^{*}$, it is easy to see that $\left(x^{*}, y^{*}\right)$ is an S-stationary point.

Conversely, assume that $\left(x^{*}, y^{*}\right)$ is S-stationary with some corresponding multipliers $\lambda, \mu, \gamma$. Then define

$$
\tilde{\gamma}_{i}:= \begin{cases}\frac{\gamma_{i}}{y_{i}^{*}} & \text { if } y_{i}^{*}>0 \\ 0 & \text { if } y_{i}^{*}=0\end{cases}
$$

The definition of S-stationarity then implies $\gamma_{i}=\tilde{\gamma}_{i} y_{i}^{*}$ for all $i=1, \ldots, n$. Therefore, setting $\delta:=0, \nu_{i}^{+}:=0, \nu_{i}^{-}:=0$ (for example), it follows immediately that $\left(x^{*}, y^{*}\right)$ together with these multipliers satisfies the above KKT conditions.

Hence S-stationarity is just a different way of writing down the KKT conditions of the relaxed problem. Note, however, that the transformation of the corresponding multipliers is not necessarily unique when going from S-stationarity to the KKT conditions. This has to be expected since the Lagrange multipliers corresponding to the KKT conditions are typically not unique (since LICQ and even MFCQ are violated), whereas the multipliers from the S-stationary conditions are obviously unique under a suitable (and obvious) linear independence assumption; see CC-LICQ below.

M-stationarity may be viewed as a slightly weaker concept than S-stationarity (as noted above) and hence a weaker optimality condition than the usual KKT conditions. More precisely, the M-stationarity conditions are exactly the KKT conditions of the following tightened nonlinear program $\operatorname{TNLP}\left(x^{*}\right)$ :

$$
\min _{x} f(x) \quad \text { s.t. } \quad g(x) \leq 0, h(x)=0, x_{i}=0\left(i \in I_{0}\left(x^{*}\right)\right) .
$$

Obviously, a local minimizer $x^{*}$ of the original problem (1.1) is also a local minimizer of $\operatorname{TNLP}\left(x^{*}\right)$ and thus an M-stationary point under suitable CQs (see below).

M-stationarity will occur in our subsequent section where it is shown that our relaxation method converges to an M-stationary point. We want to close this section with another aspect that is of some interest: S-stationarity is an optimality measure that depends both on $x$ and $y$, whereas M-stationarity depends on $x$ only. Hence 
M-stationarity may be viewed as an optimality measure of the original cardinalityconstrained problem (1.1) (which is a problem in the $x$-variables only), whereas Sstationarity involves the somewhat artificial $y$-components. In particular, this allows us to say that a vector $x^{*}$ itself (and not a pair $\left(x^{*}, y^{*}\right)$ ) is an M-stationary point of the original problem (1.1).

Let us go back to Example 4, where $\left(x^{*}, y\right)$ with any feasible $y$-component is a global solution of the relaxed problem (3.2). Applying the previous stationarity concepts, we see that $x^{*}$ is an M-stationary point. However, $\left(x^{*}, y\right)$ is S-stationary only if we pick the "right" $y$-components such as $\tilde{y}$, whereas choosing the "wrong" $y$-component such as $y^{*}$ can destroy S-stationarity.

We next want to introduce some problem-tailored CQs for the optimization problem with cardinality constraints. Again, we may try to follow the idea that our relaxed program (3.2) is closely related to MPCCs. Indeed, also for nonlinear constraints, we may assume that all variables $x_{i}$ are nonnegative. Then the relaxed program (3.2) becomes a special instance of an MPCC, and this, in principle, allows us to apply suitable MPCC-tailored constraint qualifications also to the program (3.2). However, it turns out that these MPCC-tailored conditions, though being relaxations of standard CQs, are still too strong in our case: In all feasible points $(x, y) \in Z$ with $\|x\|_{0}=\varkappa$, we have $y_{i} \in\{0,1\}$ and $\left|x_{i}\right|+y_{i} \neq 0$ for all $i=1, \ldots, n$ as well as $e^{T} y=n-\varkappa$. Thus, we have at least $n+1$ active constraints in $(x, y)$ and the corresponding gradients are $\left(0, \pm e_{i}\right)^{T}(i=1, \ldots, n)$ and $(0, e)$. This implies that MPCC-LICQ and MPCC-MFCQ are violated in all such points.

We are therefore urged to take into account the particular structure of the relaxed cardinality problem (3.2) in order to define CQs that are better suited to this program. To this end, let $\left(x^{*}, y^{*}\right)$ be a feasible point of the relaxed program (3.2), and consider again the tightened $\operatorname{NLP} \operatorname{TNLP}\left(x^{*}\right)$. We then say that $\left(x^{*}, y^{*}\right)$ satisfies a CQ for the relaxed problem (3.2) when $x^{*}$ satisfies the corresponding standard CQ for TNLP $\left(x^{*}\right)$. This leads to the following definition for CC-CPLD. (The stronger CQs CC-LICQ, CC-MFCQ, and CC-CRCQ can be defined analogously.)

DEFINITION 4.8. A point $x^{*}$ feasible for the cardinality-constrained problem (1.1) satisfies CC-CPLD if for any subsets $I_{1} \subseteq I_{g}\left(x^{*}\right), I_{2} \subseteq\{1, \ldots, p\}$, and $I_{3} \subseteq I_{0}\left(x^{*}\right)$ such that the gradients

$$
\nabla g_{i}(x)\left(i \in I_{1}\right) \quad \text { and } \quad \nabla h_{i}(x)\left(i \in I_{2}\right), e_{i}\left(i \in I_{3}\right)
$$

are positively linearly dependent in $x=x^{*}$, they are linearly dependent in a neighborhood (in $\mathbb{R}^{n}$ ) of $x^{*}$.

Thanks to the definition of these CQs via $\operatorname{TNLP}\left(x^{*}\right)$, we immediately obtain the same implications between the CC-CQs as mentioned in section 2 for standard CQs. Note that it is also possible to define suitable counterparts of ACQ and GCQ. Some details will indeed be given in a forthcoming paper, but for the purpose of this paper, these generalizations are not important.

5. Regularization method and its convergence. Having introduced the relaxed program (3.2) and taking into account its relation to the cardinality-constrained optimization problem (1.1), there exist different options for solving the original problem (1.1). One way would be to apply a branch-and-bound/cut-type strategy to the corresponding mixed-integer formulation from (3.1). This is probably the only way which guarantees finding the global optimum, but it is very costly and time-consuming and therefore not the path we want to follow here.

Alternatively, one may view the relaxed program (3.2) as an ordinary smooth 
optimization problem and apply standard software to this program. However, even in the case where $X$ is polyhedral convex, the feasible set of the relaxed program (3.2) is complicated and violates most CQs that are typically required by the existing algorithms for NLPs. Furthermore, the discussion in the previous section indicates that the standard software that tries to find KKT points may fail when $X$ is not polyhedral convex.

We therefore follow a different approach, motivated by similar considerations for mathematical programs with equilibrium constraints, and solve a sequence of suitably regularized programs with the idea that each regularized program has better properties than the relaxed program from (3.2). The particular regularization that we use here is discussed in section 5.1, and the convergence properties of the corresponding regularization method are analyzed in section 5.2. Finally, in section 5.3, we discuss some regularity properties of the regularized subproblems.

5.1. The regularized program. Here we adapt the approach from [18] and regularize the relaxed program (3.2) in the following way: Define the functions

$$
\varphi(a, b ; t):= \begin{cases}(a-t)(b-t) & \text { if } a+b \geq 2 t \\ -\frac{1}{2}\left[(a-t)^{2}+(b-t)^{2}\right] & \text { if } a+b<2 t\end{cases}
$$

as well as

$$
\tilde{\varphi}(a, b ; t):= \begin{cases}(-a-t)(b-t) & \text { if }-a+b \geq 2 t \\ -\frac{1}{2}\left[(-a-t)^{2}+(b-t)^{2}\right] & \text { if }-a+b<2 t .\end{cases}
$$

Note that $\tilde{\varphi}$ differs from the mapping $\varphi$ only in $a$ being substituted by $-a$. We want to replace the constraints $x_{i} y_{i}=0,0 \leq y_{i} \leq 1$, by the inequalities $0 \leq y_{i} \leq$ $1, \varphi\left(x_{i}, y_{i} ; t\right) \leq 0$, and $\tilde{\varphi}\left(x_{i}, y_{i} ; t\right) \leq 0$, where $t>0$ denotes a suitable parameter.

It can be easily verified that for all $t \geq 0$

$$
\varphi(a, b ; t) \leq 0 \quad \Longleftrightarrow \quad a \leq t \text { or } b \leq t \quad \Longleftrightarrow \quad \min \{a, b\} \leq t .
$$

More precisely, $\varphi(\cdot ; 0)$ is an NCP-function; see [30] for more details on such functions. Since $\tilde{\varphi}$ results from $\varphi$ by replacing $a$ with $-a$, we have for all $t \geq 0$

$$
\tilde{\varphi}(a, b ; t) \leq 0 \quad \Longleftrightarrow \quad-a \leq t \text { or } b \leq t \quad \Longleftrightarrow \quad \min \{-a, b\} \leq t .
$$

Thus, we enlarge the feasible region of the program (3.2); see Figure 4.

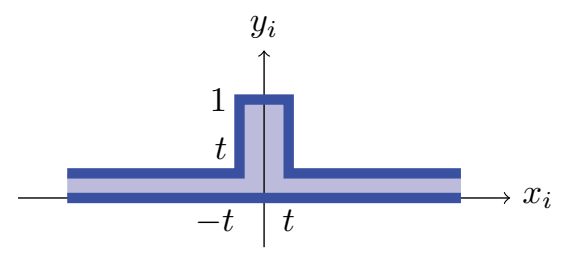

FIG. 4. Illustration of the regularized feasible set.

Similar to a result from [18], we have the following simple observation.

LEMma 5.1. The two functions $\varphi$ and $\tilde{\varphi}$ are continuously differentiable everywhere with gradients given by

$$
\nabla \varphi(a, b ; t)= \begin{cases}\left(\begin{array}{c}
b-t \\
a-t
\end{array}\right) & \text { if } a+b \geq 2 t \\
-\left(\begin{array}{c}
a-t \\
b-t
\end{array}\right) & \text { if } a+b<2 t\end{cases}
$$

Copyright (c) by SIAM. Unauthorized reproduction of this article is prohibited. 
and

$$
\nabla \tilde{\varphi}(a, b ; t)=\left\{\begin{array}{cc}
\left(\begin{array}{c}
t-b \\
-a-t
\end{array}\right) & \text { if }-a+b \geq 2 t, \\
-\left(\begin{array}{c}
a+t \\
b-t
\end{array}\right) & \text { if }-a+b<2 t,
\end{array}\right.
$$

respectively.

We now consider the following regularized problem $\operatorname{NLP}(t)$ of (3.2):

$$
\begin{array}{ll}
\min _{x, y} f(x) \quad \text { s.t. } & g_{i}(x) \leq 0 \quad \forall i=1, \ldots, m, \\
& h_{i}(x)=0 \quad \forall i=1, \ldots, p, \\
& e^{T} y \geq n-\varkappa, \\
& \varphi\left(x_{i}, y_{i} ; t\right) \leq 0 \quad \forall i=1, \ldots, n, \\
& \tilde{\varphi}\left(x_{i}, y_{i} ; t\right) \leq 0 \quad \forall i=1, \ldots, n, \\
& 0 \leq y_{i} \leq 1 \quad \forall i=1, \ldots, n,
\end{array}
$$

where $t \geq 0$ denotes a suitable parameter. Note here that, in our terminology, we distinguish between the relaxed problem (3.2) (which results from a standard relaxation of a mixed-integer problem) and the regularized problem $\operatorname{NLP}(t)$ (which, in other contexts, is also very often called a relaxation).

The regularized problem has some obvious properties which we summarize in the following result.

Proposition 5.2. Let $Z(t)$ denote the feasible set of the regularized problem $N L P(t)$, and recall that $Z$ denotes the feasible set of the relaxed program from (3.2). Then the following statements hold:

(a) $Z\left(t_{1}\right) \subseteq Z\left(t_{2}\right)$ for all $0 \leq t_{1} \leq t_{2}$.

(b) $Z \subseteq Z(t)$ for all $t \geq 0$.

(c) $Z=Z(t)$ for $t=0$.

5.2. Convergence result. The idea of the regularization method is to solve a sequence of programs $\operatorname{NLP}\left(t_{k}\right)$ with $t_{k} \downarrow 0$. Since it is unrealistic that we are able to solve (in the sense of finding a global minimum) the program $\operatorname{NLP}\left(t_{k}\right)$, we assume in the following result only that we have a sequence of KKT points, and we show that any limit point is an M-stationary point of the relaxed program (3.2) under the rather weak CC-CPLD condition. The result then, of course, also holds under the stronger LICQ- and MFCQ-type conditions.

TheOrem 5.3. Let $\left\{t_{k}\right\} \downarrow 0$ and $\left\{\left(x^{k}, y^{k}, \lambda^{k}, \mu^{k}, \delta^{k}, \tau^{k}, \tilde{\tau}^{k}, \nu^{k}\right)\right\}$ be a corresponding sequence of KKT points of $N L P\left(t_{k}\right)$ such that $\left(x^{k}, y^{k}\right) \rightarrow\left(x^{*}, y^{*}\right)$. Assume that the limit point satisfies CC-CPLD. Then $x^{*}$ is an M-stationary point of problem (3.2).

Proof. By construction of the regularization functions $\varphi$ and $\tilde{\varphi}$, the limit point $\left(x^{*}, y^{*}\right)$ is feasible for (3.2). Hence $x^{*}$ itself is feasible for the cardinality-constrained optimization problem (1.1). Furthermore, since the KKT conditions hold for each $k \in \mathbb{N}$, there exist multipliers $\lambda^{k}, \mu^{k}, \delta^{k}, \tau^{k}, \tilde{\tau}^{k}, \nu^{k}$ such that the following holds:

$$
\begin{gathered}
\nabla f\left(x^{k}\right)+\sum_{i=1}^{m} \lambda_{i}^{k} \nabla g_{i}\left(x^{k}\right)+\sum_{i=1}^{p} \mu_{i}^{k} \nabla h_{i}\left(x^{k}\right) \\
+\sum_{i=1}^{n} \tau_{i}^{k} \nabla_{x} \varphi\left(x_{i}^{k}, y_{i}^{k} ; t_{k}\right)+\sum_{i=1}^{n} \tilde{\tau}_{i}^{k} \nabla_{x} \tilde{\varphi}\left(x_{i}^{k}, y_{i}^{k} ; t_{k}\right)=0,
\end{gathered}
$$

Copyright $\odot$ by SIAM. Unauthorized reproduction of this article is prohibited. 


$$
\begin{aligned}
&-\delta^{k} e+\sum_{i=1}^{n} \tau_{i}^{k} \nabla_{y} \varphi\left(x_{i}^{k}, y_{i}^{k} ; t_{k}\right)+\sum_{i=1}^{n} \tilde{\tau}_{i}^{k} \nabla_{y} \tilde{\varphi}\left(x_{i}^{k}, y_{i}^{k} ; t_{k}\right)+\sum_{i=1}^{n} \nu_{i}^{k} e_{i}=0, \\
& \lambda_{i}^{k} \geq 0, g_{i}\left(x^{k}\right) \leq 0, \lambda_{i}^{k} g_{i}\left(x^{k}\right)=0 \quad \forall i=1, \ldots, m, \\
& h_{i}\left(x^{k}\right)=0 \quad \forall i=1, \ldots, p, \\
& \delta^{k} \geq 0, e^{T} y^{k}-n+\varkappa \geq 0, \delta^{k}\left(e^{T} y^{k}-n+\varkappa\right)=0 \\
& \tau_{i}^{k} \geq 0, \varphi\left(x_{i}^{k}, y_{i}^{k} ; t_{k}\right) \leq 0, \tau_{i}^{k} \varphi\left(x_{i}^{k}, y_{i}^{k} ; t_{k}\right)=0 \quad \forall i=1, \ldots, n, \\
& \tilde{\tau}_{i}^{k} \geq 0, \tilde{\varphi}\left(x_{i}^{k}, y_{i}^{k} ; t_{k}\right) \leq 0, \tilde{\tau}_{i}^{k} \tilde{\varphi}\left(x_{i}^{k}, y_{i}^{k} ; t_{k}\right)=0 \quad \forall i=1, \ldots, n, \\
& \nu_{i}^{k} \geq 0\left(i: y_{i}^{k}=1\right), \nu_{i}^{k}=0\left(i: y_{i}^{k} \in(0,1)\right), \nu_{i}^{k} \leq 0\left(i: y_{i}^{k}=0\right) \quad \forall i=1, \ldots, n,
\end{aligned}
$$

where $\nu_{i}^{k}$ denotes the (joint) multiplier of the box constraints $0 \leq y_{i}^{k} \leq 1$.

Using Lemma 5.1, we may rewrite the first two equations as

$$
\nabla f\left(x^{k}\right)+\sum_{i=1}^{m} \lambda_{i}^{k} \nabla g_{i}\left(x^{k}\right)+\sum_{i=1}^{p} \mu_{i}^{k} \nabla h_{i}\left(x^{k}\right)+\sum_{i=1}^{n} \tau_{i}^{k}\left(y_{i}^{k}-t_{k}\right) e_{i}+\sum_{i=1}^{n} \tilde{\tau}_{i}^{k}\left(t_{k}-y_{i}^{k}\right) e_{i}=0
$$

and

$$
\sum_{i=1}^{n} \nu_{i}^{k} e_{i}-\delta^{k} e+\sum_{i=1}^{n} \tau_{i}^{k}\left(x_{i}^{k}-t_{k}\right) e_{i}+\sum_{i=1}^{n} \tilde{\tau}_{i}^{k}\left(-x_{i}^{k}-t_{k}\right) e_{i}=0,
$$

respectively. Here, we have used the fact that we always have $\tau_{i}^{k} \nabla_{x} \varphi\left(x_{i}^{k}, y_{i}^{k} ; t_{k}\right)=$ $\tau_{i}^{k}\left(y_{i}^{k}-t_{k}\right) e_{i}$ and similarly for the other partial derivative and for the mapping $\tilde{\varphi}$. This equality comes from the observation that, if $\varphi\left(x_{i}^{k}, y_{i}^{k} ; t_{k}\right)<0$ is inactive, we have $\tau_{i}^{k}=0$ from the KKT conditions, whereas if $\varphi\left(x_{i}^{k}, y_{i}^{k} ; t_{k}\right)=0$, we necessarily have $x_{i}^{k}+y_{i}^{k} \geq 2 t_{k}$, and the equation follows from Lemma 5.1.

Now, it is easy to see that, for all $k \in \mathbb{N}$ sufficiently large, we (in particular) have

$$
\lambda_{i}^{k}>0 \quad \Longrightarrow \quad g_{i}\left(x^{k}\right)=0 \quad \Longrightarrow \quad g_{i}\left(x^{*}\right)=0
$$

and $\operatorname{supp}\left(\tau^{k}\right) \cap \operatorname{supp}\left(\tilde{\tau}^{k}\right)=\emptyset$. The latter implies that the multipliers

$$
\gamma_{i}^{k}:= \begin{cases}\tau_{i}^{k}\left(y_{i}^{k}-t_{k}\right) & \text { if } i \in \operatorname{supp}\left(\tau^{k}\right), \\ \tilde{\tau}_{i}^{k}\left(t_{k}-y_{i}^{k}\right) & \text { if } i \in \operatorname{supp}\left(\tilde{\tau}^{k}\right), \\ 0 & \text { otherwise }\end{cases}
$$

are well defined and, by (5.1), satisfy

$$
\nabla f\left(x^{k}\right)+\sum_{i=1}^{m} \lambda_{i}^{k} \nabla g_{i}\left(x^{k}\right)+\sum_{i=1}^{p} \mu_{i}^{k} \nabla h_{i}\left(x^{k}\right)+\sum_{i=1}^{n} \gamma_{i}^{k} e_{i}=0 .
$$

We claim that, for all $i$ with $x_{i}^{*} \neq 0$, we have $\gamma_{i}^{k}=0$ for all $k \in \mathbb{N}$ sufficiently large. First, consider the case $x_{i}^{*}>0$. Then $x_{i}^{k}>t_{k}$ for all $k$ sufficiently large. If $i \in \operatorname{supp}\left(\tau^{k}\right)$, the KKT conditions imply $\varphi\left(x_{i}^{k}, y_{i}^{k} ; t_{k}\right)=0$, and therefore, in view of the definition of this mapping, we necessarily get $y_{i}^{k}=t_{k}$ which, in turn, yields $\gamma_{i}^{k}=0$. On the other hand, if $i \in \operatorname{supp}\left(\tilde{\tau}^{k}\right)$, we have $\tilde{\varphi}\left(x_{i}^{k}, y_{i}^{k} ; t_{k}\right)=0$; hence once again $y_{i}^{k}=t_{k}$ since $-x_{i}^{k}-t_{k}<0$ for all sufficiently large $k$. This also yields $\gamma_{i}^{k}=0$. For $i \notin \operatorname{supp}\left(\tau^{k}\right) \cup \operatorname{supp}\left(\tilde{\tau}^{k}\right)$, we automatically have $\gamma_{i}^{k}=0$ by definition. In a similar way, one can treat the case $x_{i}^{*}<0$, which implies $-x_{i}^{k}>t_{k}$ for all $k$ sufficiently large, and the corresponding arguments are then symmetric to the case $x_{i}^{*}>0$. 
By [29, Lemma A.1], we can assume without loss of generality that the gradients (including the unit vectors) corresponding to nonvanishing multipliers in (5.2) are linearly independent. Note that this might change the multipliers $\left\{\left(\lambda^{k}, \mu^{k}, \gamma^{k}\right)\right\}$ but preserves their signs, and vanishing multipliers remain zero.

We claim that the sequence $\left\{\left(\lambda^{k}, \mu^{k}, \gamma^{k}\right)\right\}$ is bounded. Assume it is unbounded. Taking a subsequence if necessary, we may assume without loss of generality that the corresponding normalized sequence converges, say

$$
\frac{\left(\lambda^{k}, \mu^{k}, \gamma^{k}\right)}{\left\|\left(\lambda^{k}, \mu^{k}, \gamma^{k}\right)\right\|_{2}} \rightarrow(\bar{\lambda}, \bar{\mu}, \bar{\gamma}) \neq 0
$$

Dividing (5.2) by $\left\|\left(\lambda^{k}, \mu^{k}, \gamma^{k}\right)\right\|$ and taking the limit $k \rightarrow \infty$, we then obtain

$$
\sum_{i=1}^{m} \bar{\lambda}_{i} \nabla g_{i}\left(x^{*}\right)+\sum_{i=1}^{p} \bar{\mu}_{i} \nabla h_{i}\left(x^{*}\right)+\sum_{i=1}^{n} \bar{\gamma}_{i} e_{i}=0
$$

with $\bar{\lambda}_{i} \geq 0$ for all $i=1, \ldots, m$ and $\bar{\lambda}_{i}=0$ for all $i$ such that $g_{i}\left(x^{*}\right)<0$ (since then $g_{i}\left(x^{k}\right)<0$ for all $k$ sufficiently large and, therefore, $\lambda_{i}^{k}=0$ in view of the corresponding KKT conditions). Furthermore, for all $i$ with $x_{i}^{*} \neq 0$, we have $\gamma_{i}^{k}=0$ for all $k$ sufficiently large in view of the preceding discussion and, therefore, also $\bar{\gamma}_{i}=0$. Hence, we know $\bar{\lambda} \geq 0, \operatorname{supp}(\bar{\lambda}) \subseteq I_{g}\left(x^{*}\right)$, and $\operatorname{supp}(\bar{\gamma}) \subseteq I_{0}\left(x^{*}\right)$. But then by CC-CPLD, the positively linearly dependent gradients

$$
\left\{\nabla g_{i}\left(x^{*}\right) \mid i \in \operatorname{supp}(\bar{\lambda})\right\} \cup\left\{\left\{\nabla h_{i}\left(x^{*}\right) \mid i \in \operatorname{supp}(\bar{\mu})\right\} \cup\left\{e_{i} \mid i \in \operatorname{supp}(\bar{\gamma})\right\}\right\}
$$

would have to remain linearly dependent in a neighborhood of $x^{*}$, a contradiction to the choice of the multipliers $\left\{\left(\lambda^{k}, \mu^{k}, \gamma^{k}\right)\right\}$.

This shows that the sequence $\left\{\left(\lambda^{k}, \mu^{k}, \gamma^{k}\right)\right\}$ remains bounded. Subsequencing if necessary, we may therefore assume that $\left(\lambda^{k}, \mu^{k}, \gamma^{k}\right) \rightarrow(\lambda, \mu, \gamma)$. Similar to the previous argument, we then obtain

$$
\begin{array}{r}
\nabla f\left(x^{*}\right)+\sum_{i=1}^{m} \lambda_{i} \nabla g_{i}\left(x^{*}\right)+\sum_{i=1}^{p} \mu_{i} \nabla h_{i}\left(x^{*}\right)+\sum_{i=1}^{n} \gamma_{i} e_{i}=0, \\
\lambda_{i} \geq 0\left(i \in I_{g}\left(x^{*}\right)\right), \quad \lambda_{i}=0\left(i \notin I_{g}\left(x^{*}\right)\right), \\
\gamma_{i}=0\left(i: x_{i}^{*} \neq 0\right)
\end{array}
$$

i.e., $x^{*}$ is an M-stationary point.

5.3. Properties of the regularized subproblems. Since we want to solve the regularized problems $\operatorname{NLP}\left(t_{k}\right)$ numerically, it would be beneficial to know whether they inherit properties such as CQs from the original relaxed problem (3.2). In order to answer this question, we define the following index sets for a $t>0$ and $(\hat{x}, \hat{y})$ 
feasible for $\operatorname{NLP}(t)$ :

$$
\begin{aligned}
I_{\varphi}(\hat{x}, \hat{y} ; t) & :=\left\{i \in\{1, \ldots, n\} \mid \varphi\left(\hat{x}_{i}, \hat{y}_{i} ; t\right)=0\right\}, \\
I_{\varphi}^{00}(\hat{x}, \hat{y} ; t) & :=\left\{i \in\{1, \ldots, n\} \mid \hat{x}_{i}=t, \hat{y}_{i}=t\right\}, \\
I_{\varphi}^{0+}(\hat{x}, \hat{y} ; t) & :=\left\{i \in\{1, \ldots, n\} \mid \hat{x}_{i}=t, \hat{y}_{i}>t\right\}, \\
I_{\varphi}^{+0}(\hat{x}, \hat{y} ; t) & :=\left\{i \in\{1, \ldots, n\} \mid \hat{x}_{i}>t, \hat{y}_{i}=t\right\}, \\
I_{\tilde{\varphi}}(\hat{x}, \hat{y} ; t) & :=\left\{i \in\{1, \ldots, n\} \mid \tilde{\varphi}\left(\hat{x}_{i}, \hat{y}_{i} ; t\right)=0\right\}, \\
I_{\tilde{\varphi}}^{00}(\hat{x}, \hat{y} ; t) & :=\left\{i \in\{1, \ldots, n\} \mid \hat{x}_{i}=-t, \hat{y}_{i}=t\right\}, \\
I_{\tilde{\varphi}}^{0+}(\hat{x}, \hat{y} ; t) & :=\left\{i \in\{1, \ldots, n\} \mid \hat{x}_{i}=-t, \hat{y}_{i}>t\right\}, \\
I_{\tilde{\varphi}}^{-0}(\hat{x}, \hat{y} ; t) & :=\left\{i \in\{1, \ldots, n\} \mid \hat{x}_{i}<-t, \hat{y}_{i}=t\right\} .
\end{aligned}
$$

Note that, due to the feasibility of $(\hat{x}, \hat{y})$, the three index sets $I_{\varphi}^{00}(\hat{x}, \hat{y} ; t), I_{\varphi}^{0+}(\hat{x}, \hat{y} ; t)$, and $I_{\varphi}^{+0}(\hat{x}, \hat{y} ; t)$ form a partitioning of the set $I_{\varphi}(\hat{x}, \hat{y} ; t)$. A corresponding observation holds for the index set $I_{\tilde{\varphi}}(\hat{x}, \hat{y} ; t)$.

For all subsets $I \subseteq I_{\varphi}^{00}(\hat{x}, \hat{y} ; t)$ and $\tilde{I} \subseteq I_{\tilde{\varphi}}^{00}(\hat{x}, \hat{y} ; t)$, we define the $\operatorname{NLPs} \operatorname{NLP}(t, I, \tilde{I})$ as

$$
\begin{array}{rl}
\min _{x, y} & f(x) \quad \text { s.t. } \quad g(x) \leq 0, h(x)=0, e^{T} y \geq n-\varkappa, \\
& 0 \leq y_{i} \leq t \forall i \in I_{\varphi}^{+0}(\hat{x}, \hat{y} ; t) \cup\left(I_{\varphi}^{00}(\hat{x}, \hat{y} ; t) \backslash I\right) \cup I_{\tilde{\varphi}}^{-0}(\hat{x}, \hat{y} ; t) \cup\left(I_{\tilde{\varphi}}^{00}(\hat{x}, \hat{y} ; t) \backslash \tilde{I}\right), \\
& -t \leq x_{i} \leq t, 0 \leq y_{i} \leq 1 \quad \forall i \in I_{\varphi}^{0+}(\hat{x}, \hat{y} ; t) \cup I \cup I_{\tilde{\varphi}}^{0+}(\hat{x}, \hat{y} ; t) \cup \tilde{I} \\
& \varphi\left(x_{i}, y_{i} ; t\right) \leq 0, \tilde{\varphi}\left(x_{i}, y_{i} ; t\right) \leq 0,0 \leq y_{i} \leq 1, \quad \forall i \notin I_{\varphi}(\hat{x}, \hat{y} ; t) \cup I_{\tilde{\varphi}}(\hat{x}, \hat{y} ; t) .
\end{array}
$$

Let us denote the feasible set of $\operatorname{NLP}(t)$ by $Z(t)$, and the feasible set of $\operatorname{NLP}(t, I, \tilde{I})$ by $Z(t, I, \tilde{I})$. Analogously to Proposition 4.1 , one can show that $(\hat{x}, \hat{y}) \in Z(t, I, \tilde{I})$ for all subsets $I \subseteq I_{\varphi}^{00}(\hat{x}, \hat{y} ; t)$ and $\tilde{I} \subseteq I_{\tilde{\varphi}}^{00}(\hat{x}, \hat{y} ; t)$. Furthermore, there exists a sufficiently small $r>0$ such that

$$
Z(t) \cap B_{r}(\hat{x}, \hat{y})=\left(\bigcup_{I \subseteq I_{\varphi}^{00}(\hat{x}, \hat{y}, t), \tilde{I} \subseteq I_{\tilde{\varphi}}^{00}(\hat{x}, \hat{y} ; t)} Z(t, I, \tilde{I})\right) \cap B_{r}(\hat{x}, \hat{y})
$$

holds. In fact, due to the preceding observation, it is easy to see that the right-hand side is included in the left-hand side, and the other direction follows by taking, e.g.,

$$
I:=\left\{i \in I_{\varphi}^{00}(\hat{x}, \hat{y} ; t) \mid y_{i}>t\right\} \quad \text { and } \quad \tilde{I}:=\left\{i \in I_{\tilde{\varphi}}^{00}(\hat{x}, \hat{y} ; t) \mid y_{i}>t\right\} .
$$

Similar to Lemma 4.2, this implies

$$
\begin{aligned}
T_{Z(t)}(\hat{x}, \hat{y}) & =\bigcup_{I \subseteq I_{\varphi}^{00}(\hat{x}, \hat{y} ; t), \tilde{I} \subseteq I_{\dot{\varphi}}^{00}(\hat{x}, \hat{y} ; t)} T_{Z(t, I, \tilde{I})}(\hat{x}, \hat{y}), \\
T_{Z(t)}(\hat{x}, \hat{y})^{*} & =\bigcap_{I \subseteq I_{\varphi}^{00}(\hat{x}, \hat{y} ; t), \tilde{I} \subseteq I_{\tilde{\varphi}}^{00}(\hat{x}, \hat{y} ; t)} T_{Z(t, I, \tilde{I})}(\hat{x}, \hat{y})^{*} .
\end{aligned}
$$

Using these preparations, we can now prove the main result in this section.

THEOREM 5.4. Let $\left(x^{*}, y^{*}\right)$ be feasible for the relaxed problem (3.2). When $C C$ CPLD is satisfied in $\left(x^{*}, y^{*}\right)$, then there is a $\bar{t}>0$ and an $r>0$ such that the 
following holds for all $t \in(0, \bar{t}]:$ If $(\hat{x}, \hat{y}) \in B_{r}\left(x^{*}\right) \times B_{r}\left(y^{*}\right)$, is feasible for $N L P(t)$, then standard GCQ for $N L P(t)$ holds there.

Proof. Since CC-CPLD holds in $\left(x^{*}, y^{*}\right)$ and the constraints are continuously differentiable, there is a neighborhood $B_{r}\left(x^{*}\right)$ such that the gradients

$$
\left\{\nabla g_{i}(x) \mid i \in I_{g}\left(x^{*}\right)\right\} \cup\left\{\left\{\nabla h_{j}(x) \mid j=1, \ldots, p\right\} \cup\left\{e_{i} \mid i \in I_{0}\left(x^{*}\right)\right\}\right\}
$$

satisfy CPLD in every element $\hat{x} \in B_{r}\left(x^{*}\right)$; i.e., all subsets of these gradients, which are positively linearly dependent at $\hat{x}$, remain linearly dependent in a neighborhood of $\hat{x}$. Decreasing $r>0$ if necessary, we can find a $\bar{t}>0$ such that for all $t \in(0, \bar{t}]$ all elements $(\hat{x}, \hat{y}) \in B_{r}\left(x^{*}\right) \times B_{r}\left(y^{*}\right)$ feasible for $\operatorname{NLP}(t)$ additionally satisfy

$$
I_{g}(\hat{x}) \subseteq I_{g}\left(x^{*}\right) \quad \text { and } \quad I_{\varphi}^{0+}(\hat{x}, \hat{y}, t) \cup I_{\varphi}^{00}(\hat{x}, \hat{y}, t) \cup I_{\tilde{\varphi}}^{0+}(\hat{x}, \hat{y}, t) \cup I_{\tilde{\varphi}}^{00}(\hat{x}, \hat{y}, t) \subseteq I_{0}\left(x^{*}\right) .
$$

Now consider an arbitrary $t \in(0, \hat{t}]$ and an arbitrary $(\hat{x}, \hat{y}) \in B_{r}\left(x^{*}\right) \times B_{r}\left(y^{*}\right)$ feasible for $\operatorname{NLP}(t)$. The point $(\hat{x}, \hat{y})$ is then feasible for all $\operatorname{NLP}(t, I, \tilde{I})$ with $I \subseteq I_{\varphi}^{00}(\hat{x}, \hat{y} ; t)$ and $\tilde{I} \subseteq I_{\tilde{\varphi}}^{00}(\hat{x}, \hat{y} ; t)$ (see the discussion preceding this theorem), and the gradients of the active inequality constraints and the equality constraints at that point are

$$
\begin{aligned}
& \left\{\left\{\left(\begin{array}{c}
\nabla g_{i}(\hat{x}) \\
0
\end{array}\right) \mid i \in I_{g}(\hat{x})\right\} \cup\left\{\left(\begin{array}{c}
0 \\
-e
\end{array}\right) \mid \text { if } e^{T} \hat{y}=n-\varkappa\right\}\right. \\
& \cup\left\{\left(\begin{array}{c}
0 \\
e_{i}
\end{array}\right) \mid i \in I_{\varphi}^{+0}(\hat{x}, \hat{y} ; t) \cup\left(I_{\varphi}^{00}(\hat{x}, \hat{y} ; t) \backslash I\right) \cup I_{\tilde{\varphi}}^{-0}(\hat{x}, \hat{y} ; t) \cup\left(I_{\tilde{\varphi}}^{00}(\hat{x}, \hat{y} ; t) \backslash \tilde{I}\right)\right\} \\
& \cup\left\{\left(\begin{array}{c}
e_{i} \\
0
\end{array}\right) \mid i \in I_{\varphi}^{0+}(\hat{x}, \hat{y} ; t) \cup I\right\} \cup\left\{\left(\begin{array}{c}
-e_{i} \\
0
\end{array}\right) \mid i \in I_{\tilde{\varphi}}^{0+}(\hat{x}, \hat{y} ; t) \cup \tilde{I}\right\} \\
& \left.\cup\left\{\left(\begin{array}{c}
0 \\
e_{i}
\end{array}\right) \mid \hat{y}_{i}=1\right\} \cup\left\{\left(\begin{array}{c}
0 \\
-e_{i}
\end{array}\right) \mid \hat{y}_{i}=0\right\}\right\} \\
& \cup\left\{\left(\begin{array}{c}
\nabla h_{j}(\hat{x}) \\
0
\end{array}\right) \mid j=1, \ldots, p\right\} .
\end{aligned}
$$

Since all constraints depend either on $x$ or on $y$ but never on both, we can show that $\operatorname{CPLD}$ for $\operatorname{NLP}(t, I, \tilde{I})$ is satisfied at $(\hat{x}, \hat{y})$ by considering them separately. All constraints depending on $y$ are linear and therefore satisfy the CPLD condition. The constraints depending on $x$, in turn, satisfy CPLD due to the choice of $r$ and $\bar{t}$.

Since CPLD implies ACQ (cf. section 2), we thus have shown that

$$
T_{Z(t, I, \tilde{I})}(\hat{x}, \hat{y})=L_{Z(t, I, \tilde{I})}(\hat{x}, \hat{y})
$$

holds for all $I \subseteq I_{\varphi}^{00}(\hat{x}, \hat{y} ; t)$ and $\tilde{I} \subseteq I_{\tilde{\varphi}}^{00}(\hat{x}, \hat{y} ; t)$. Combining this with (5.4), we obtain

$$
T_{Z(t)}(\hat{x}, \hat{y})^{*}=\bigcap_{I \subseteq I_{\varphi}^{00}(\hat{x}, \hat{y} ; t), \tilde{I} \subseteq I_{\tilde{\varphi}}^{00}(\hat{x}, \hat{y} ; t)} L_{Z(t, I, \tilde{I})}(\hat{x}, \hat{y})^{*} .
$$

In order to prove that $(\hat{x}, \hat{y})$ satisfies GCQ for $\operatorname{NLP}(t)$, we have to prove the inclusion $T_{Z(t)}(\hat{x}, \hat{y})^{*} \subseteq L_{Z(t)}(\hat{x}, \hat{y})^{*}$. Hence our next step is to calculate the linearization cones 
and their polar cones. For $\operatorname{NLP}(t)$, these are (cf. Lemma 5.1)

$$
\begin{aligned}
& L_{Z(t)}(\hat{x}, \hat{y})=\left\{\left(d_{x}, d_{y}\right) \in \mathbb{R}^{n} \times \mathbb{R}^{n} \mid \forall_{i \in I_{g}(\hat{x})} \quad \nabla g_{i}(\hat{x})^{T} d_{x} \leq 0,\right. \\
& \forall i=1, \ldots, p \quad \nabla h_{i}(\hat{x})^{T} d_{x}=0,
\end{aligned}
$$

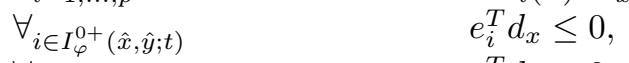

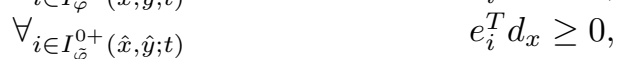

$$
\begin{aligned}
& \text { if } e^{T} \hat{y}=n-\varkappa \quad e^{T} d_{y} \geq 0, \\
& \forall_{i \in\left\{i \mid \hat{y}_{i}=1\right\} \cup I_{\varphi}^{+0}(\hat{x}, \hat{y} ; t) \cup I_{\tilde{\varphi}}^{-0}(\hat{x}, \hat{y} ; t)} e_{i}^{T} d_{y} \leq 0 \\
& \left.\forall i \in\left\{i \mid \hat{y}_{i}=0\right\} \quad e_{i}^{T} d_{y} \geq 0\right\}
\end{aligned}
$$

and

$$
\begin{aligned}
& L_{Z(t)}(\hat{x}, \hat{y})^{*}=\left\{\left(w_{x}, w_{y}\right) \in \mathbb{R}^{n} \times \mathbb{R}^{n} \mid w_{x}=\sum_{i \in I_{g}(\hat{x})} \lambda_{i} \nabla g_{i}(\hat{x})+\sum_{i=1}^{p} \mu_{i} \nabla h_{i}(\hat{x})+\sum_{i=1}^{n} \gamma_{i} e_{i},\right. \\
& w_{y}=\delta e+\sum_{i=1}^{n} \nu_{i} e_{i}, \\
& \begin{array}{ll}
\forall i \in I_{g}(\hat{x}) & \lambda_{i} \geq 0,
\end{array} \\
& \forall_{i \in I_{\varphi}^{0+}(\hat{x}, \hat{y} ; t) \quad \gamma_{i} \geq 0,}
\end{aligned}
$$

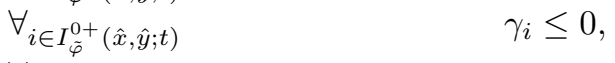

$$
\begin{aligned}
& \forall \text { other } i \quad \gamma_{i}=0, \\
& \delta \leq 0 \text { and } \delta=0 \quad \text { if } e^{T} \hat{y}>n-\varkappa \text {, }
\end{aligned}
$$

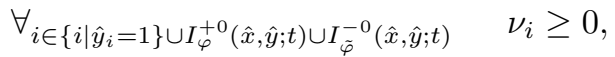

$$
\begin{aligned}
& \forall_{i \in\left\{i \mid \hat{y}_{i}=0\right\}} \quad \nu_{i} \leq 0 \\
& \left.\forall \nu_{i}=0\right\} \text {. }
\end{aligned}
$$

For $\operatorname{NLP}(t, I, \tilde{I})$ the cones are

$$
\begin{aligned}
& L_{Z(t, I, \tilde{I})}(\hat{x}, \hat{y})=\left\{\left(d_{x}, d_{y}\right) \in \mathbb{R}^{n} \times \mathbb{R}^{n} \mid \forall_{i \in I_{g}(\hat{x})}\right. \\
& \forall i=1, \ldots, p \\
& \forall_{i \in I_{\varphi}^{0+}(\hat{x}, \hat{y} ; t) \cup I} \\
& \forall_{i \in I_{\tilde{\varphi}}^{0+}(\hat{x}, \hat{y} ; t) \cup \tilde{I}} \\
& \text { if } e^{T} \hat{y}=n-\varkappa \quad e^{T} d_{y} \geq 0, \\
& \forall_{i \in\left\{i \mid \hat{y}_{i}=1\right\} \cup I_{\varphi}^{+0}(\hat{x}, \hat{y} ; t) \cup\left(I_{\varphi}^{00}(\hat{x}, \hat{y} ; t) \backslash I\right)} e_{i}^{T} d_{y} \leq 0, \\
& \forall_{i \in \cup I_{\tilde{\varphi}}^{-0}(\hat{x}, \hat{y} ; t) \cup\left(I_{\tilde{\varphi}}^{00}(\hat{x}, \hat{y} ; t) \backslash \tilde{I}\right)} \quad e_{i}^{T} d_{y} \leq 0, \\
& \forall i \in\left\{i \mid \hat{y}_{i}=0\right\} \\
& \nabla g_{i}(\hat{x})^{T} d_{x} \leq 0 \\
& \nabla h_{i}(\hat{x})^{T} d_{x}=0 \\
& e_{i}^{T} d_{x} \leq 0 \\
& e_{i}^{T} d_{x} \geq 0 \\
& \left.e_{i}^{T} d_{y} \geq 0\right\}
\end{aligned}
$$

and

$$
\begin{aligned}
& L_{Z(t, I, \tilde{I})}(\hat{x}, \hat{y})^{*}=\left\{\left(w_{x}, w_{y}\right) \in \mathbb{R}^{n} \times \mathbb{R}^{n} \mid w_{x}=\sum_{i \in I_{g}(\hat{x})} \lambda_{i} \nabla g_{i}(\hat{x})+\sum_{i=1}^{p} \mu_{i} \nabla h_{i}(\hat{x})+\sum_{i=1}^{n} \gamma_{i} e_{i},\right. \\
& w_{y}=\delta e+\sum_{i=1}^{n} \nu_{i} e_{i} \\
& \forall_{i \in I_{g}(\hat{x})} \quad \lambda_{i} \geq 0, \\
& \forall_{i \in I_{\varphi}^{0+}(\hat{x}, \hat{y} ; t) \cup I} \quad \gamma_{i} \geq 0, \\
& \forall_{i \in I_{\tilde{\varphi}}^{0+}(\hat{x}, \hat{y} ; t) \cup \tilde{I}} \quad \gamma_{i} \leq 0, \\
& \forall \text { other } i \quad \gamma_{i}=0 \text {, } \\
& \delta \leq 0 \text { and } \delta=0 \quad \text { if } e^{T} \hat{y}>n-\varkappa \text {, }
\end{aligned}
$$

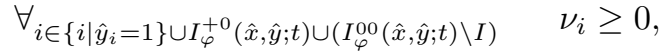

$$
\begin{aligned}
& \forall_{i \in I_{\tilde{\varphi}}^{-0}(\hat{x}, \hat{y} ; t) \cup\left(I_{\tilde{\varphi}}^{00}(\hat{x}, \hat{y} ; t) \backslash \tilde{I}\right)} \quad \nu_{i} \geq 0, \\
& \forall i \in\left\{i \mid \hat{y}_{i}=0\right\} \quad \nu_{i} \leq 0, \\
& \begin{aligned}
\forall \text { other } i & \left.\nu_{i}=0\right\} .
\end{aligned}
\end{aligned}
$$

Copyright $@$ by SIAM. Unauthorized reproduction of this article is prohibited. 
We now put everything together: Let $\left(w_{x}, w_{y}\right) \in T_{Z(t)}(\hat{x}, \hat{y})^{*}$ be arbitrarily given. In view of (5.5), this implies that the point $\left(w_{x}, w_{y}\right)$ also belongs to $L_{Z(t, \emptyset, \emptyset)}(\hat{x}, \hat{y})^{*}$ and $L_{Z\left(t, I_{\varphi}^{00}(\hat{x}, \hat{y} ; t), I_{\varphi}^{00}(\hat{x}, \hat{y} ; t)\right)}(\hat{x}, \hat{y})^{*}$. Taking into account that $\gamma_{i}$ and $\nu_{i}$ only occur separately in the expressions for $w_{x}$ and $w_{y}$, respectively, this immediately gives $\left(w_{x}, w_{y}\right) \in$ $L_{Z(t)}(\hat{x}, \hat{y})^{*}$. Together, this proves that GCQ for $\operatorname{NLP}(t)$ is satisfied at $(\hat{x}, \hat{y})$.

Note that, in order to obtain a similar result for the related regularization method for MPCCs, an LICQ-type condition had to be assumed in [18], whereas here only CC-CPLD is required.

6. Numerical results. To test the approach presented in this paper, we consider cardinality-constrained problems of the form

$$
\begin{aligned}
\min _{x} x^{T} Q x \quad \text { s.t. } & \mu^{T} x \geq \rho \\
& e^{T} x \leq 1 \\
& 0 \leq x_{i} \leq u_{i} \quad \forall i=1, \ldots, n, \\
& \|x\|_{0} \leq \varkappa .
\end{aligned}
$$

This is a classical portfolio optimization problem where $Q$ and $\mu$ are the covariance matrix and mean of $n$ possible assets, respectively, and $e^{T} x \leq 1$ is a resource constraint; see, e.g., $[6,10]$. To create test examples, we take the same randomly generated data $Q, \mu, \rho$, and $u$ which were used by Frangioni and Gentile in [13] and which are available at their Webpage [14]. This gives us 30 test instances for each of the three dimensions $n=200,300,400$. In addition, we consider for every instance the three cardinality constraints defined by $\varkappa=5,10,20$ and thus end up with 270 test problems.

We implemented the following three solution strategies in MATLAB: First, we followed [6] (see also Remark 2, where $y_{i}$ was replaced by $1-y_{i}$ for an easier comparison with our approach) and reformulated the cardinality-constrained problem using binary constraints as

$$
\begin{array}{ccc}
\min _{x, y} x^{T} Q x \quad \text { s.t. } & \mu^{T} x \geq \rho, & \\
& e^{T} x \leq 1, & \\
& 0 \leq x_{i} \leq u_{i} y_{i} \quad \forall i=1, \ldots, n, \\
& y_{i} \in\{0,1\} & \forall i=1, \ldots, n, \\
& e^{T} y \leq \varkappa . &
\end{array}
$$

We tried to solve these mixed-integer problems directly using GUROBI 5.6.2 via the provided MATLAB interface. GUROBI is a solver specialized in mixed-integer linear and quadratic optimization problems (see [16]). To avoid serious memory problems experienced earlier, we set the parameter MIPFocus $=1$ for GUROBI to spend more effort on finding good feasible solutions quickly and less effort on proving optimality. Additionally, we set TimeLimit $=600$ to limit the calculation time to 10 minutes. This may sound very restrictive, but in our numerical experiments we observed that GUROBI most often found a good solution within the first 60 seconds and then spent the remaining time on proving optimality. All computations were performed on a hyperthreading-enabled computer with six cores, so the 600 seconds correspond to approximately two hours of computation time. 
Our second approach is based on the relaxed problem (3.2), which in this case is

$$
\begin{aligned}
\min _{x, y} x^{T} Q x \quad \text { s.t. } & \mu^{T} x \geq \rho, \\
& e^{T} x \leq 1 \\
& 0 \leq x_{i} \leq u_{i} \quad \forall i=1, \ldots, n, \\
& e^{T} y \geq n-\varkappa \\
& x_{i} y_{i}=0 \quad \forall i=1, \ldots, n, \\
& 0 \leq y_{i} \leq 1 \quad \forall i=1, \ldots, n .
\end{aligned}
$$

This problem has orthogonality/complementarity-type constraints. Since it can still be viewed as a standard nonlinear optimization problem, we applied the TOMLAB version of SNOPT to solving (6.1). SNOPT is based on an SQP approach combined with an augmented Lagrangian merit function [15].

Finally, we implemented the regularization method from the previous section as well. It replaces the orthogonality condition $x_{i} y_{i}=0$ with the two inequalities $\varphi\left(x_{i}, y_{i} ; t\right) \leq 0$ and $\tilde{\varphi}\left(x_{i}, y_{i} ; t\right) \leq 0$. Due to the presence of the constraint $x_{i} \geq 0$ in our test problems, we could ignore the inequality $\tilde{\varphi}\left(x_{i}, y_{i} ; t\right) \leq 0$. Nonetheless, it is still included to make the implementation applicable to more general instances. We solved the regularized problems $\mathrm{NLP}(t)$ iteratively using the TOMLAB version of SNOPT, beginning with the regularization parameter $t_{0}=1$. In every iteration, we decreased the regularization parameter by $t_{k+1}=0.01 t_{k}$ and used the solution of the previous iteration as our initial value. We stopped the algorithm if either the regularization parameter became too small, i.e., $t_{k}<10^{-8}$, or the violation of the orthogonality conditions was sufficiently small, i.e., $\max _{i=1, \ldots, n}\left|x_{i} y_{i}\right| \leq 10^{-6}$. The feasibility of the other constraints (all of which are linear) never caused any problems.

We used $x^{0}=(0, \ldots, 0)^{T}$ and $y^{0}=(1, \ldots, 1)^{T}$ as initial values for all three methods. In the following, the computational results are grouped by $n$ and $\varkappa$. The average computation time in seconds and the average orthogonality violation can be found in Table 1 . Here, the orthogonality violation means $\max _{i=1, \ldots, n}\left|x_{i}\left(1-y_{i}\right)\right|$ for GUROBI and $\max _{i=1, \ldots, n}\left|x_{i} y_{i}\right|$ for the other two approaches. Since the violations of the linear and box constraints are, if existent, a lot smaller than the violation of the orthogonality constraint, we choose not to display them.

TABle 1

Average computation time $T$ and average orthogonality violation $v$.

\begin{tabular}{lc|ccc|ccc|ccc}
\hline & $n$ & \multicolumn{3}{c|}{200} & \multicolumn{3}{c|}{300} & \multicolumn{3}{c}{400} \\
& $x$ & 5 & 10 & 20 & 5 & 10 & 20 & 5 & 10 & 20 \\
\hline \multirow{2}{*}{ GUROBI } & $T$ & 600.2 & 600.2 & 600.1 & 580.8 & 598.4 & 580.8 & 596.8 & 600.1 & 600.1 \\
& $v$ & 0 & 0 & 0 & 0 & 0 & 0 & 0 & 0 & 0 \\
\hline \multirow{2}{*}{ Relaxation } & $T$ & 0.0608 & 0.0592 & 0.0551 & 0.1743 & 0.1587 & 0.1089 & 0.2499 & 0.2129 & 0.1981 \\
& $v$ & $10^{-12}$ & $10^{-12}$ & $10^{-12}$ & $2 \cdot 10^{-12}$ & $2 \cdot 10^{-12}$ & $2 \cdot 10^{-12}$ & $3 \cdot 10^{-12}$ & $3 \cdot 10^{-12}$ & $3 \cdot 10^{-12}$ \\
\hline \multirow{2}{*}{ Regularization } & $T$ & 1.3562 & 1.4810 & 1.9636 & 3.3107 & 3.6135 & 3.8653 & 7.0481 & 7.3284 & 8.0956 \\
& $v$ & $10^{-6}$ & $10^{-6}$ & $10^{-6}$ & $10^{-6}$ & $10^{-6}$ & $10^{-6}$ & $10^{-6}$ & $10^{-6}$ & $10^{-6}$ \\
\hline
\end{tabular}

Figure 5 illustrates the different objective function values found by the three methods. For every test example, we divided the values found by all three methods by that found by GUROBI and plotted the resulting factors; hence the GUROBI lines are normalized to 1 . Thus, a value of 10 for the relaxed approach would mean that, for this example, the relaxed approach found a solution where the objective function value was 10 times as big as that found by GUROBI. The order in which the results for the 30 test examples are plotted for each $n$ and $\varkappa$ is chosen such that the normalized 

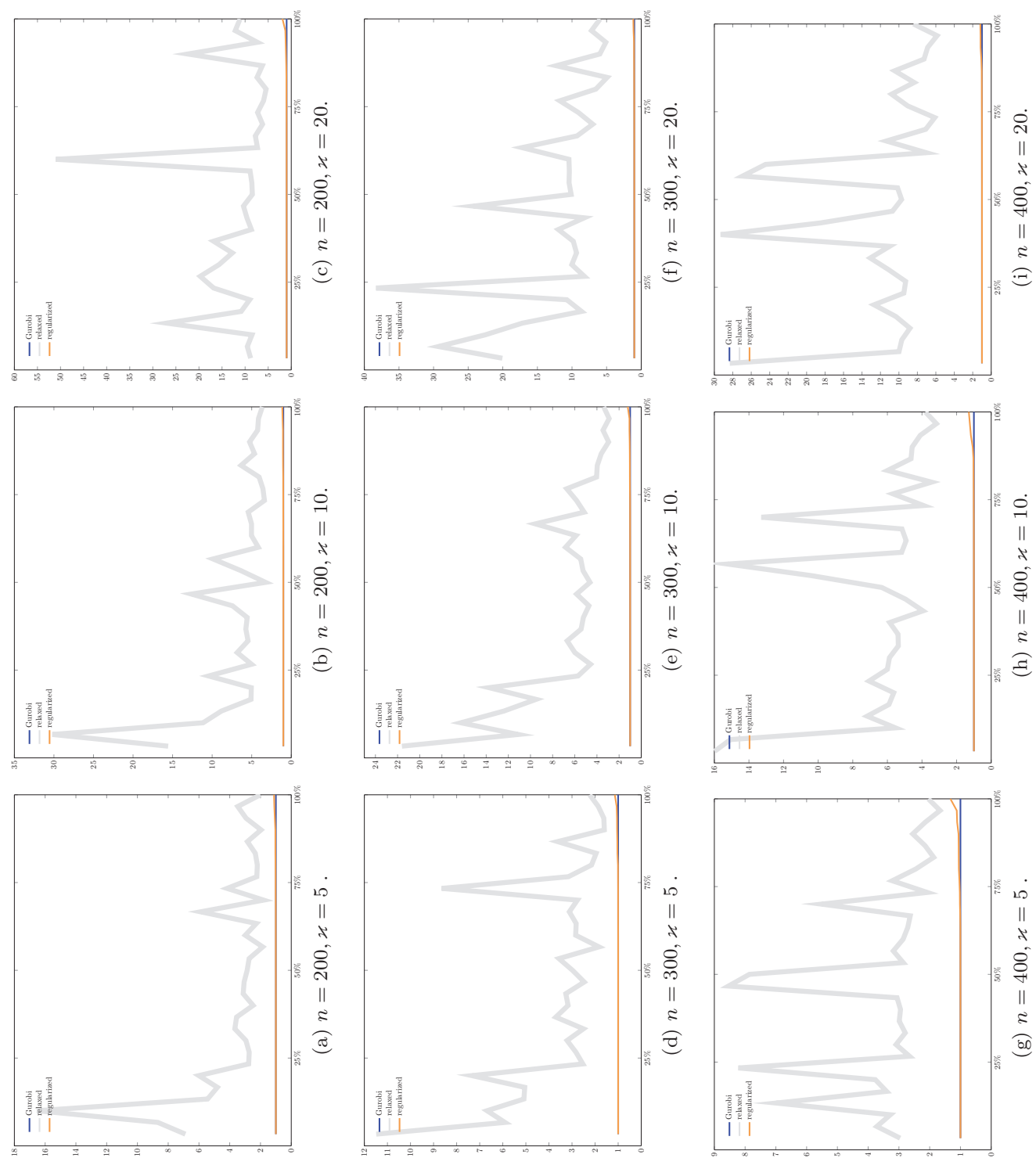

FIG. 5. Normalized objective function values for all examples.

values obtained for the regularization method are ascending. This way it is easy to see that, e.g., for $n=200$ and $\varkappa=20$, the regularization method obtains function values almost equal to those found by GUROBI in more than $90 \%$ of the considered problems. More detailed results for each test run can be found in the tables given in the appendix of the preprint version of this paper [8].

If we compare the average computation times, we see that the relaxed approach is the fastest, followed by the regularized method. Whenever the average computation time of GUROBI is less than 600 seconds, GUROBI managed to solve one of the 30 test examples in less than 10 minutes.

The orthogonality constraints also hold. Due to the declaration of $y_{i}$ as a binary variable, GUROBI produces no measurable violation of the orthogonality. The slightly 
higher orthogonality violation of the regularization method compared to the relaxation approach is a direct consequence of the fact that we terminated the regularization method as soon as this violation was at most $10^{-6}$. Nonetheless, if we consider the solutions found by the regularization method and eliminate the $\varkappa$ largest components, the remaining entries, which should be zero, exceed $10^{-6}$ in only 1 out of 270 examples.

The most interesting results are the objective function values in the solutions. Even though we allowed GUROBI to run only for 600 seconds, it managed to find the best value in almost all examples. However, when we compare the relaxation and regularization approaches, we see a huge difference. Whereas the value found by the relaxation approach is always significantly worse than that found by GUROBI (in more than $80 \%$ of the examples between 2.5 and 14 times as big), the regularization method performs almost as well as GUROBI. In $71.5 \%$ of the examples, it manages to find a similar (in the sense that the function value is at most $1 \%$ worse than the one computed by GUROBI) or even slightly better value than does GUROBI. In all test examples, the value is less than 2 times as big as GUROBI's. Hence, although neither method in its present implementation can completely keep up with the commercial (global!) solver GUROBI, the regularization method shows very promising performance, whereas relaxing the cardinality-constrained problem without additional regularization seems less successful.

One possible reason for the better results of the regularization method is that the regularized feasible set is bigger than that of the relaxed problem, and therefore the method is probably less dependent on the initial value. In order to illustrate this effect, we consider the problem

$$
\min _{x} x_{1}+10 x_{2} \quad \text { s.t. } \quad\left(x_{1}-\frac{1}{2}\right)^{2}+\left(x_{2}-1\right)^{2} \leq 1, \quad\|x\|_{0} \leq 1 .
$$

The feasible set then consists of a part of the $x_{2}$-axis including the local minimizer $\left(0,1-\frac{1}{2} \sqrt{3}\right)^{T}$ and the isolated global minimizer $\left(\frac{1}{2}, 0\right)^{T}$. We discretized the rectangle $\left[-1, \frac{3}{2}\right] \times\left[-\frac{1}{2}, 2\right]$ and started both the relaxation approach and the regularization method in each of the resulting 441 node points. As expected, the regularization method converged to the global minimizer in all 441 cases, whereas the relaxation approach found the global solution only in 204 cases, which is less than $50 \%$.

7. Final remarks. This paper presents an NLP-formulation of the cardinalityconstrained optimization problem. Several theoretical results regarding minima and stationary points as well as a suitable regularization method are presented. We believe that the NLP-reformulation given here can be used as the basis for several other developments for cardinality-constrained problems. Here we mention only two points that will be part of our future research: First, instead of extending the regularization scheme from [18] to cardinality constraints, it seems more obvious to apply the regularization from [28] to cardinality constraints. To some extent, this has been done in the context of sparse optimization problems in [12], for a simple example. We tried to apply this approach for more general problems but have failed so far due to some complications with certain Lagrange multipliers. Second, taking into account that we derived the NLP-reformulation as a relaxation of a mixed-integer problem, there should be a good chance to use techniques from binary problems in order to get closer to a global minimum.

Acknowledgment. We would like to thank two anonymous referees for carefully reading our work and for their helpful suggestions. 


\section{REFERENCES}

[1] R. Andreani, J. M. Martínez, and M. L. Schuverdt, The CPLD condition of Qi and Wei implies the quasinormality qualification, J. Optim. Theory Appl., 125 (2005), pp. 473-485.

[2] M. S. Bazaraa and C. M. Shetty, Foundations of Optimization, Lecture Notes in Econ. Math. Syst., Springer, New York, 1976.

[3] A. BeCK AND Y. C. EldAR, Sparsity constrained nonlinear optimization: Optimality conditions and algorithms, SIAM J. Optim., 23 (2013), pp. 1480-1509.

[4] D. P. Bertsekas And A. E. Ozdaglar, Pseudonormality and a Lagrange multiplier theory for constrained optimization, J. Optim. Theory Appl., 114 (2002), pp. 287-343.

[5] D. Bertsimas and R. Shioda, Algorithm for cardinality-constrained quadratic optimization, Comput. Optim. Appl., 43 (2009), pp. 1-22.

[6] D. Bienstock, Computational study of a family of mixed-integer quadratic programming problems, Math. Programming, 74 (1996), pp. 121-140.

[7] O. Burdakov, C. Kanzow, And A. Schwartz, On a reformulation of mathematical programs with cardinality constraints, in Advances in Global Optimization, D. Y. Gao, N. Ruan, and W. X. Xing, eds., Proceedings of the 3rd World Congress of Global Optimization (Huangshan, China, 2013), Springer, New York, 2015, pp. 3-14.

[8] O. Burdakov, C. Kanzow, And A. Schwartz, Mathematical Programs with Cardinality Constraints: Reformulation by Complementarity-type Conditions and a Regularization Method, Preprint 324, Institute of Mathematics, University of Würzburg, Würzburg, Germany, 2014.

[9] E. J. CANDÈS AND M. B. WAKIn, An introduction to compressive sampling, IEEE Trans. Signal Process., 25 (2008), pp. 21-30.

[10] D. Di Lorenzo, G. Liuzzi, F. Rinaldi, F. Schoen, And M. Sciandrone, A concave optimization-based approach for sparse portfolio selection, Optim. Methods Softw., 27 (2012), pp. 983-1000.

[11] F. FACChinei AND J.-S. PANG, Finite-Dimensional Variational Inequalities and Complementarity Problems, Volumes I and II, Springer Ser. Oper. Res., Springer, New York, 2003.

[12] M. Feng, J. E. Mitchell, J.-S. PAng, X. Shen, And A. Wächter, Complementarity Formulation of $\ell_{0}$-norm Optimization Problems, Technical Report, Industrial Engineering and Management Sciences, Northwestern University, Evanston, IL, 2013.

[13] A. Frangioni and C. Gentile, SDP diagonalizations and perspective cuts for a class of nonseparable MIQP, Oper. Res. Lett., 35 (2007), pp. 181-185.

[14] A. Frangioni And C. Gentile, Mean-variance problem with minimum buy-in constraints, data and documentation, online at http://www.di.unipi.it/optimize/Data/MV.html.

[15] P. E. Gill, W. Murray, and M. A. Saunders, SNOPT: An SQP algorithm for large-scale constrained optimization, SIAM J. Optim., 12 (2002), pp. 979-1006.

[16] Gurobi Optimization, Gurobi Optimizer Reference Manual, http://www.gurobi.com, 2014.

[17] R. JANin, Directional derivative of the marginal function in nonlinear programming, Math. Program. Stud., 21 (1984), pp. 110-126.

[18] C. Kanzow And A. Schwartz, A new regularization method for mathematical programs with complementarity constraints with strong convergence properties, SIAM J. Optim., 23 (2013), pp. 770-798.

[19] Z.-Q. LuO, J.-S. PANG, AND D. RAlPh, Mathematical Programs with Equilibrium Constraints, Cambridge University Press, Cambridge/New York/Melbourne, 1996.

[20] A. Miller, Subset Selection in Regression, 2nd ed., Chapman \& Hall/CRC Press, Boca Raton, FL, 2002.

[21] W. Murray and H. Shek, A local relaxation method for the cardinality constrained portfolio optimization problem, Comput. Optim. Appl., 53 (2012), pp. 681-709.

[22] J. Nocedal And S. J. Wright, Numerical Optimization, Springer Ser. Oper. Res., Springer, New York, 1999.

[23] J. V. Outrata, M. KoČvara, and J. Zowe, Nonsmooth Approach to Optimization Problems with Equilibrium Constraints, Kluwer Academic Publishers, Dordrecht, The Netherlands, 1998.

[24] L. QI AND Z. WEI, On the constant positive linear dependence condition and its application to SQP methods, SIAM J. Optim., 10 (2000), pp. 963-981.

[25] R. T. Rockafellar and R. J.-B. Wets: Variational Analysis, Grundlehren Math. Wiss. 317, Springer, New York, 1998.

[26] R. Ruiz-Torrubiano, S. García-Moratilla, and A. SuÁrez, Optimization problems with cardinality constraints, in Computational Intelligence in Optimization, Y. Tenne and C.K. Goh, eds., Springer, New York, 2010, pp. 105-130. 
[27] H. Scheel And S. Scholtes, Mathematical programs with complementarity constraints: Stationarity, optimality, and sensitivity, Math. Oper. Res., 25 (2000), pp. 1-22.

[28] S. SCHOLTES, Convergence properties of a regularization scheme for mathematical programs with complementarity constraints, SIAM J. Optim., 11 (2001), pp. 918-936.

[29] S. Steffensen and M. Ulbrich, A new relaxation scheme for mathematical programs with equilibrium constraints, SIAM J. Optim., 20 (2010), pp. 2504-2539.

[30] D. Sun And L. QI, On NCP-functions, Comput. Optim. Appl., 13 (1999), pp. 201-220.

[31] X. Sun, X. Zheng, AND D. LI, Recent advances in mathematical programming with semicontinuous variables and cardinality constraint, J. Oper. Res. Soc. China, 1 (2013), pp. 5577.

[32] X. Zheng, X. Sun, D. Li, And J. Sun, Successive convex approximations to cardinalityconstrained convex programs: A piecewise-linear DC approach, Comput. Optim. Appl., to appear. DOI: $10.1007 / \mathrm{s} 10589-013-9582-3$.

Copyright (c) by SIAM. Unauthorized reproduction of this article is prohibited. 